\title{
NEW EXAMPLES OF OBSTRUCTIONS TO NON-NEGATIVE SECTIONAL CURVATURES IN COHOMOGENEITY ONE MANIFOLDS
}

\begin{abstract}
CHENXU HE
Abstract. K. Grove, L. Verdiani, B. Wilking and W. Ziller gave the first examples of cohomogeneity one manifolds which do not carry invariant metrics with non-negative sectional curvatures. In this paper we generalize their results to a larger family. We also classify all class one representations for a pair $(G, H)$ with $G / H$ a sphere, which are used to construct the examples.
\end{abstract}

\section{INTRODUCTION}

Non-negatively curved Riemannian manifolds have been of interest from the beginning of global Riemannian geometry. Most examples are obtained via product and quotient constructions, starting from compact Lie groups. These include all homogeneous spaces and biquotients. Using a gluing method, J. Cheeger constructed non-negatively curved metrics on the connect sum of any two rank one symmetric spaces; see [Ch]. A breakthrough came with K. Grove and W. Ziller's generalization of this gluing method to the class of cohomogeneity one manifolds.

A compact manifold $M$ is called a cohomogeneity one manifold if there exists a compact Lie group $G$ acting on $M$ by isometries, and the cohomogeneity of the action, defined as $\operatorname{cohom}(M, G)=\operatorname{dim}(M / G)$, is equal to 1 . Since the orbit space is one dimensional, it is either a circle or a closed interval $I$. In the former case, $M$ always carries a $G$ invariant metric with non-negative sectional curvature. In the latter, there are precisely two singular orbits $B_{ \pm}$with isotropy subgroups $K^{ \pm}$ corresponding to the endpoints of $I$, a minimal geodesic between the singular orbits, and principal orbits corresponding to the interior points with isotropy subgroup $H$. By the slice theorem, $K^{ \pm} / H$ are spheres $\mathbb{S}^{l_{ \pm}-1}\left(l_{ \pm} \geq 1\right)$, and $M$ can be reconstructed by gluing two disk bundles along a principal orbit as follows:

$$
M=G \times_{K^{-}} \mathbb{D}^{l_{-}} \bigcup_{G / H} G \times_{K^{+}} \mathbb{D}^{l_{+}},
$$

where $\mathbb{D}^{l_{ \pm}}$is the normal disk to $B_{ \pm}$. Therefore we can identify $M$ with the groups $H \subset\left\{K^{-}, K^{+}\right\} \subset G$ by the gluing construction (1.1).

K. Grove and W. Ziller showed that any cohomogeneity one manifold with codimension two singular orbits admits a non-negatively curved metric [GZ1]. In the same paper, it was conjectured that any cohomogeneity one manifold admits a non-negatively curved metric. This turns out to be false. The first examples of an obstruction were discovered by K. Grove, L. Verdiani, B. Wilking and W. Ziller in GVWZ. The most interesting examples are the higher dimensional Kervaire

Received by the editors June 21, 2012 and, in revised form, April 8, 2013.

2010 Mathematics Subject Classification. Primary 53C20, 53C30.

(C)2014 American Mathematical Society Reverts to public domain 28 years from publication 
spheres (of dimension 9 and up) which are the only exotic spheres that can carry a cohomogeneity one action; see $\mathrm{St}$.

In light of the construction of examples with non-negative sectional curvature and the examples of obstructions to non-negatively curved metrics, it is important to answer the question "How large is the class of cohomogeneity one manifolds that admit a non-negatively curved metric?" which was raised by W. Ziller in [Zi].

In this paper, we generalize the examples in [GVWZ] to a larger family:

Theorem. Let $K^{\prime} / H^{\prime}=\mathbb{S}^{k}$ with $k \geq 2$ and $\rho: K^{\prime} \longrightarrow S O(m)$ be a faithful irreducible representation, which is not the one of $K^{\prime}$ on $\mathbb{R}^{k+1}$, and such that $\rho\left(H^{\prime}\right) \subset S O(m-1)$. For any integer $n \geq m+2$, set $G=S O(n)$ and

$$
\begin{aligned}
K^{-} & =\rho\left(K^{\prime}\right) \times S O(n-m) \subset S O(m) \times S O(n-m) \subset S O(n), \\
K^{+} & =\rho\left(H^{\prime}\right) \times S O(n-m+1) \subset S O(m-1) \times S O(n-m+1) \subset S O(n), \\
H & =\rho\left(H^{\prime}\right) \times S O(n-m) \subset S O(n) .
\end{aligned}
$$

Then the cohomogeneity one manifold $M$ defined by the groups $H \subset\left\{K^{-}, K^{+}\right\} \subset G$ does not admit a $G$ invariant metric with non-negative sectional curvature.

In GVWZ, the Theorem was proved under the additional assumptions that the slice representation of $K^{\prime}$ is not contained in the symmetric square $\operatorname{Sym}^{2} \rho$ and $\rho\left(K^{\prime}\right)$ does not act transitively on the sphere $\mathbb{S}^{m-1}=S O(m) / S O(m-1)$.

This Theorem is optimal in the sense that if $\rho$ is the representation of $K^{\prime}$ on $\mathbb{R}^{k+1}$, then the manifold $M$ does admit an invariant non-negatively curved metric, since it is diffeomorphic to the homogeneous space $S O(n+1) /\left(\rho\left(K^{\prime}\right) \times S O(n-m+1)\right)$ endowed with the cohomogeneity one action of $S O(n) \subset S O(n+1)$. On the other hand, if $n=m+1$, one obtains an interesting cohomogeneity one manifold where it is not known whether or not that manifold carries a non-negatively curved invariant metric.

The Theorem can be extended to the case where $\rho$ is not necessarily irreducible; see Theorem 3.1. A representation $\rho: K^{\prime} \longrightarrow S O(m)$ with $\rho\left(H^{\prime}\right) \subset S O(m-1)$ is called a class one representation; see Definition 2.12. Many class one representations are not faithful (see Proposition A.3 and Table 2), so they are excluded by the faithfulness requirement in [GVWZ. However Theorem 3.1 allows non-faithful class one representation as a subrepresentation of $\rho$ and it gives us many more examples; see section 3 ,

Similar results also hold if $\rho$ is a complex or quaternionic representation, i.e., $G=U(n)$ or $S p(n)$, which are stated in Theorem 6.1 and Theorem 6.6.

In the above Theorem, Theorem 3.1 Theorem 6.1 and Theorem 6.6. the group $K^{\prime}$ does not need to be connected. But the corresponding groups $K^{ \pm}$on the universal cover $\widetilde{M}$ will be connected. Therefore in the rest of the paper we can assume that all groups are connected.

The paper is organized as follows. In section 2, we briefly recall basic properties of cohomogeneity one manifolds, the generalized Weyl group and metric properties. Also this section includes an introduction to class one representations. A more detailed discussion of such representations is given in Appendix A. In section 3 we prove some basic properties of the new examples which will be used in the proof of the orthogonal case. In section 4, we study the consequences of the nonnegativity assumption on the metrics. Section 5 contains the proof of the orthogonal case. Using properties of the metrics developed in the previous sections, we draw a 
contradiction by looking at sectional curvatures of two distinct classes of 2-planes. In section 6 , we sketch an outline of the proofs in the case where $G=U(n)$ or $S p(n)$.

\section{Preliminaries}

In this section, we recall some basic and well-known facts about closed cohomogeneity one manifolds. For more details, we refer to, for example, $\underline{\mathrm{AA}}$ and GWZ.

We know that there are precisely two non-principal orbits $B_{ \pm}$in a simply connected cohomogeneity one manifold. Suppose $M$ is endowed with an invariant metric $g$ and the distance between the two non-principal orbits is $L$. Let $c(t), t \in \mathbb{R}$, be a geodesic minimizing the distance with $c(0)=p_{-} \in B_{-}$and $c(L)=p_{+} \in B_{+}$. The isotropy subgroups at $p_{ \pm}$are denoted by $K^{ \pm}$and the principal isotropy subgroup at any point $c(t), t \in(0, L)$, is denoted by $H$. The group diagram $H \subset\left\{K^{-}, K^{+}\right\} \subset G$ is not uniquely determined by the manifold since one can switch $K^{-}$with $K^{+}$, change $g$ to another invariant metric and choose another minimal geodesic $c(t)$.

Definition 2.1. Two group diagrams are called equivalent if they determine the same cohomogeneity one manifold up to equivariant diffeomorphism.

The following lemma characterizes which two group diagrams are equivalent; see in GWZ, p. 44].

Lemma 2.2. Two group diagrams $H \subset\left\{K^{-}, K^{+}\right\} \subset G$ and $\tilde{H} \subset\left\{\tilde{K}^{-}, \tilde{K}^{+}\right\} \subset G$ are equivalent if and only if after possibly switching the roles of $K^{-}$and $K^{+}$, the following holds: There exist elements $b \in G$ and $a \in N(H)_{0}$, where $N(H)_{0}$ is the identity component of the normalizer of $H$, with $\tilde{K}^{-}=b K^{-} b^{-1}, \tilde{H}=b H b^{-1}$, and $\tilde{K}^{+}=a b K^{+} b^{-1} a^{-1}$.

Remark 2.3. If $c(t)$ is the minimal normal geodesic between the two non-principal orbits, then $b \star c(t)$ is another minimal geodesic between $B_{ \pm}$, and the associated group diagram is obtained by conjugating all isotropy groups by the element $b$. We can assume that $b \in N(H) \cap N\left(K^{-}\right)$in order to fix $H$ and $K^{-}$. Conjugation by an element $a$ as in the above lemma usually corresponds to changing the invariant metric on the manifold.

Let $C \subset M$ be the image of the minimal geodesic $c(t)$. Then the Weyl group $\mathcal{W}$ of the $G$-action on $M$ is by definition the stabilizer of $C$ modulo its kernel $H$. $\mathcal{W}$ is characterized in the following proposition.

Proposition 2.4. The Weyl group $\mathcal{W}$ of a cohomogeneity one manifold is a dihedral subgroup of $N(H) / H$. It is generated by involutions $w_{ \pm} \in\left(N(H) \cap K^{ \pm}\right) / H$ and $C / \mathcal{W}=M / G=[0, L]$. Each of these involutions can be represented as an element $a \in K^{ \pm} \cap H$ such that $a^{2}$ but not a lies in $H$.

Using the group action, the invariant metric is determined by its restriction to the minimal geodesic $c(t)$. Suppose $c(t)$ is parameterized by arc length, i.e., $T=\frac{\mathrm{d}}{\mathrm{d} t}$ has length 1 . Then we can write $g$ as

$$
g=\mathrm{d} t^{2}+g_{t}
$$

and $\left\{g_{t}\right\}_{t \in[0, L]}$ is a one-parameter family of homogeneous metrics on the orbits $M_{t}=G \cdot c(t)$. 
Fix a bi-invariant inner product $Q$ on the Lie algebra $\mathfrak{g}$ of $G$ and let $\mathfrak{p}=\mathfrak{h}^{\perp}$ be the orthogonal complement of the Lie algebra $\mathfrak{h}$ of $H$. For each $X \in \mathfrak{p}$, let $X^{*}$ be the Killing vector field generated by $X$ along $c(t)$, i.e., $X^{*}(t)=\left.\frac{\mathrm{d}}{\mathrm{d} s}\right|_{s=0} \exp (s X) . c(t)$. For each $t \in(0, L), M_{t}$ is diffeomorphic to the homogeneous space $G / H$, and hence $T_{c(t)} M_{t}$ can be identified with $\mathfrak{p}$ by means of Killing vector fields as $X \mapsto X^{*}(t)$. Then $g_{t}$ defines an inner product on $\mathfrak{p}$ which is invariant under the isotropy action of $\operatorname{Ad}_{H}$. We set

$$
g_{t}(X, Y)=g_{t}\left(X^{*}(t), Y^{*}(t)\right)=Q\left(P_{t} X, Y\right) \text { for } X, Y \in \mathfrak{p},
$$

where $P_{t}: \mathfrak{p} \longrightarrow \mathfrak{p}$ is a $Q$-symmetric $\mathrm{Ad}_{H}$-equivariant endomorphism. The metric $g$ is completely determined by the one-parameter family $\left\{P_{t}\right\}, t \in[0, L]$, and at $t=0$ and $L, P_{t}$ should satisfy further conditions to guarantee smoothness of $g$.

On the other hand, each principal orbit $M_{t}$ is a hypersurface in $M$ with normal vector $T$. If $S_{t} X=S_{t} X^{*}(t)=-\nabla_{X^{*}} T$ is the shape operator of $M_{t}$ at $c(t)$, we have

$$
S_{t}=-\frac{1}{2} P_{t}^{-1} P_{t}^{\prime}
$$

in terms of $P$.

In the rest of this section, we give a short introduction to class one representations, with more details in Appendix A, This particular class of representations is well studied; see, for example, $\mathrm{VK}$ ] and $\mathrm{Wa}$.

Definition 2.7. A representation $(\mu, W)$ of a compact Lie group $K$ is called a real (complex or quaternionic) representation if $W$ is a vector space over $\mathbb{R}(\mathbb{C}$ or $\mathbb{H})$.

Definition 2.8. Suppose $W$ is an irreducible representation over the complex numbers; then $W$ is called a real representation or of real type if it comes from a representation over reals by extension of scalars. It is of quaternionic type if it comes from a representation over quaternions by restriction of scalars. It is of complex type if it is neither real or quaternionic.

For any complex representation $\mu$, let $\mu^{*}$ denote its complex conjugate. $\mu^{*}$ is equivalent to $\mu$ if $\mu$ is of type real or quaternionic and they are non-equivalent if $\mu$ is of complex type. Suppose $K$ is a compact connected Lie group; then the complexification of a real irreducible representation $\sigma$ is one of the following:

(1) $\sigma_{\mathbb{C}}=\mu$, where $\mu$ is an irreducible representation of real type,

(2) $\sigma_{\mathbb{C}}=\mu \oplus \mu^{*}$, where $\mu$ is irreducible and of complex type,

(3) $\sigma_{\mathbb{C}}=\mu \oplus \mu$, where $\mu$ is irreducible and of quaternionic type.

In classes (2) and (3), we often write $\sigma=[\mu]_{\mathbb{R}}$.

Conversely, suppose $\mu$ is a complex irreducible representation of $K$ with degree $n$. If $\mu$ is of real type, then there exists a real vector space $\mathbb{R}^{n} \subset \mathbb{C}^{n}$ which is $\mu(K)$ invariant. Let $\sigma$ be the restriction of $\mu$ on $\mathbb{R}^{n}$; then $\sigma$ is a real irreducible representation with $\sigma_{\mathbb{C}}=\mu$. If $\mu$ is not of real type, then we identify $\mathbb{C}^{n}$ with $\mathbb{R}^{2 n}$ and forget the complex structure on it. Thus $\mathbb{R}^{2 n}$ is invariant under the $\mu(K)$ action and it gives us a real irreducible representation. We denote this representation by $\sigma$, and then $\sigma_{\mathbb{C}}$ is either $\mu \oplus \mu^{*}$ or $\mu \oplus \mu$ depending on the type of $\mu$.

A quaternionic irreducible representation is obtained by extending the scalar field to the quaternions for a complex irreducible representation, and the converse also holds.

Given an $n$ dimensional real irreducible representation $(\sigma, W)$ of a compact Lie group $K$, we briefly discuss the classification of the equivariant endomorphisms, 
i.e., the endomorphism $f: W \longrightarrow W$ with $f(\sigma(g) \cdot v)=\sigma(g) \cdot f(v)$ for any $g \in K$ and $v \in W$.

If $\sigma$ is in class (1), then from Schur's lemma, $f=a \operatorname{Id}$ for some constant $a \in \mathbb{R}$ and Id is the identity map of $W$.

If $\sigma$ is in class (2), then $W$ has an orthonormal basis such that $f$ is in the following form under that basis:

$$
f=\left(\begin{array}{cc}
a_{0} I_{m} & -a_{1} I_{m} \\
a_{1} I_{m} & a_{0} I_{m}
\end{array}\right),
$$

where $a_{0}, a_{1}$ are constants in $\mathbb{R}, n=2 m$ and $I_{m}$ is the $m \times m$ identity matrix.

If $\sigma$ is in class (3), then there exists an orthonormal basis of $W$ such that $f$ has the following form under that basis:

$$
f=\left(\begin{array}{cccc}
a_{0} I_{m} & -a_{1} I_{m} & -a_{2} I_{m} & -a_{3} I_{m} \\
a_{1} I_{m} & a_{0} I_{m} & a_{3} I_{m} & -a_{2} I_{m} \\
a_{2} I_{m} & -a_{3} I_{m} & a_{0} I_{m} & a_{1} I_{m} \\
a_{3} I_{m} & a_{2} I_{m} & -a_{1} I_{m} & a_{0} I_{m}
\end{array}\right),
$$

where $n=4 m, a_{0}, a_{1}, a_{2}$ and $a_{3}$ are constants in $\mathbb{R}$.

Definition 2.11. A pair $(K, H)$ of compact Lie groups with $H \subset K$ and $K / H=\mathbb{S}^{k}$ $(k \geq 2)$ is called a spherical pair.

If we assume that $K$ is connected, the image of $\mu$ will be a closed subgroup of $S O(l), U(l)$ or $S p(l)$ if $\mu$ is over $\mathbb{R}, \mathbb{C}$ or $\mathbb{H}$.

For each group pair $(K, H)$ with $H \subset K$ a closed subgroup, we have

Definition 2.12. A non-trivial irreducible representation $(\mu, W)$ of $K$ is called $a$ class one representation of the pair $(K, H)$ if $\mu(H)$ fixes a non-zero vector $w_{0} \in W$.

Remark 2.13. From Proposition A.3, the class one representations of spherical pairs are almost effective, i.e., the kernel of $\mu$ is discrete, except for the following cases:

(1) $(K, H)=\left(U(n), U(n-1)_{m}\right)$ and $\mu=a e_{1}-a e_{n}$, where $a$ is a positive integer. The kernel is the diagonal embedded $U(1) \subset U(n)$.

(2) $(S p(n) \times S p(1), S p(n-1) \times S p(1))$ and $\mu=a \varpi_{2}$, where $a$ is a positive integer. The kernel contains the $S p(1)$ factor.

(3) $\left(S p(n) \times U(1), S p(n-1) \times U(1)_{m}\right)$ and $\mu=\left(a \varpi_{1}+b \varpi_{2}\right) \otimes$ id, where $a, b$ are non-negative integers with $a+b \geq 1$ and id is the trivial representation of $U(1)$. The kernel contains the $U(1)$ factor.

In the case where $(K, H)=(S O(k+1), S O(k))$, let $\varpi_{1}$ be the highest weight of the standard representation $\varrho_{k+1}$ on $\mathbb{R}^{k+1}$. Then the class one representations over $\mathbb{R}$ are precisely those with the highest weights as $m \varpi_{1}, m=1,2, \ldots$. These representation spaces can be realized as the space of homogeneous harmonic polynomials. Let $\left\{x_{1}, \ldots, x_{k+1}\right\}$ be the basis of $\mathbb{R}^{k+1}$ and $S O(k+1)$ act by the matrix multiplication. Then an element $A \in S O(k+1)$ acts on a polynomial $f\left(x_{1}, \ldots, x_{k+1}\right)$ through the action on the variables, i.e., $(A . f)\left(x_{1}, \ldots, x_{k+1}\right)=f\left(A^{-1} \cdot x_{1}, \ldots, A^{-1} \cdot x_{k+1}\right)$. Let $H_{m}$ be the space of homogeneous harmonic polynomials in $x_{1}, \ldots, x_{k+1}$ of degree $m$; then the representation of $S O(k+1)$ on $H_{m}$ has the highest weight $m \varpi_{1}$. All of them are of real type. If $k+1$ is odd, then the representation for any positive $m$ is faithful. If $k+1$ is even, then the class one representation is faithful if and only if $m$ is odd. As we will see, the class one representations of other spherical 
pairs $(K, H)$ are the irreducible components when $H_{m}$ is restricted to the subgroup $K$ of $S O(k+1)$.

The real, complex and quaternionic class one representations of the spherical pairs are classified in Appendix A. Further properties are discussed there as well.

\section{WEYL GROUP AND SMOOTHNESS}

First let us state the most general result in the orthogonal case as a generalization of the theorem in the introduction using the concept of class one representation:

Theorem 3.1. Let $K^{\prime} / H^{\prime}=\mathbb{S}^{k}$ with $k \geq 2$ and $\rho: K^{\prime} \longrightarrow S O(m)$ be a faithful representation that contains a (not necessarily faithful) irreducible class one representation $\mu$ of the pair $\left(K^{\prime}, H^{\prime}\right)$ such that one of the following holds:

(1) $\operatorname{deg} \mu \geq k+2$ if $\mu$ is of real type or if the multiplicity of $\mu$ in $\rho$, denoted by $\operatorname{mul}(\mu, \rho)$, is equal to 1 ;

(2) $\operatorname{deg} \mu \geq 2(k+2)$ if $\mu$ is of complex type and $\operatorname{mul}(\mu, \rho) \geq 2$;

(3) $\operatorname{deg} \mu \geq 4(k+2)$ if $\mu$ is of quaternionic type and $\operatorname{mul}(\mu, \rho) \geq 2$.

We assume that $n \geq m+2$ if $\operatorname{mul}(\mu, \rho)=1$, and that $n \geq m+3$ if $\operatorname{mul}(\mu, \rho) \geq 2$. If we set $G=S O(n)$ and

$$
\begin{aligned}
K^{-} & =\rho\left(K^{\prime}\right) \times S O(n-m) \subset S O(m) \times S O(n-m) \subset S O(n), \\
K^{+} & =\rho\left(H^{\prime}\right) \times S O(n-m+1) \subset S O(m-1) \times S O(n-m+1) \subset S O(n), \\
H & =\rho\left(H^{\prime}\right) \times S O(n-m) \subset S O(n),
\end{aligned}
$$

then the cohomogeneity one manifold $M$ defined by the groups $H \subset\left\{K^{-}, K^{+}\right\} \subset G$ does not admit a $G$ invariant metric with non-negative sectional curvature.

Remark 3.3. Since we do not assume that $\mu$ is faithful, a non-faithful class one representation is allowed in this construction. In other words, if $\mu$ is a class one representation with $\operatorname{deg} \mu \geq k+2$, we choose a representation $\tau$ with $\operatorname{ker} \tau \cap \operatorname{ker} \mu=$ $\{1\}$. Then $\rho=\tau \oplus \mu$ satisfies the conditions in the theorem. For example, take $\left(K^{\prime}, H^{\prime}\right)=(S O(6), S O(5))$ with $k=5$ and let $\mu$ be the 20 dimensional representation of $S O(6)$ with the highest weight $2 \varpi_{1}$. To construct a cohomogeneity one manifold, we can choose $\tau=\varrho_{6}$ as the standard representation of $S O(6)$ which is faithful and let $\rho=\tau \oplus \mu$.

Remark 3.4. If $\rho$ contains only one copy of $\mu$ or $\mu$ is of real type, then Table 3 in Proposition A.4 lists all real class one representations which have dimensions smaller than $k+2$. We see that only the following representations are excluded by the assumption $\operatorname{deg} \mu \geq k+2$ : the defining representation of $K^{\prime}$ on $\mathbb{R}^{k+1}$, the 9 dimensional representation $\varrho_{9}$ of the pair $(\operatorname{Spin}(9), \operatorname{Spin}(7))$, the 5 dimensional representation of $(S p(2), S p(1))$ and the 3 dimensional representations of $(S U(2),\{\operatorname{Id}\})$ and $(U(2), U(1))$. All of these representations are not faithful, so one needs to add another representation $\tau$ with $\operatorname{ker} \tau \cap \operatorname{ker} \mu=\{\operatorname{Id}\}$ to define a cohomogeneity one manifold. For such manifolds, we do not know if they admit an invariant metric with non-negative curvature.

Remark 3.5. If $\rho$ contains more than one copy of $\mu$ and $\mu$ is not of real type, then the further restriction $\operatorname{deg} \mu \geq 2(k+2)$ or $4(k+2)$ excludes 8 more representations as listed in the last part of Table [3. Among them, the following representations are faithful: $\mu=\left[2 \varpi_{1}\right]_{\mathbb{R}}$ for the pair $(S U(3), S U(2)), \mu=\left[\varpi_{1}+\varpi_{2}\right]_{\mathbb{R}}$ for the pair $(S p(2), S p(1))$ and $\mu=\left[3 \varpi_{1}\right]_{\mathbb{R}},\left[5 \varpi_{1}\right]_{\mathbb{R}}$ and $\left[7 \varpi_{1}\right]_{\mathbb{R}}$ for the pair $(S p(1),\{1\})$. The 
first one is of complex type and the other four are of quaternionic type. They can be used to construct cohomogeneity one manifolds without adding other representations; for example, take $\left(K^{\prime}, H^{\prime}\right)=(S p(1),\{1\})$ and $\rho=\left[3 \varpi_{1}\right]_{\mathbb{R}} \oplus\left[3 \varpi_{1}\right]_{\mathbb{R}}$. Thus Theorem 3.1 does not give obstruction for such manifolds.

Remark 3.6. The lowest dimensional example of Theorem 3.1 is obtained as follows. Take $\left(K^{\prime}, H^{\prime}\right)=(S O(3), S O(2))$ with $K^{\prime} / H^{\prime}=\mathbb{S}^{2}$. Then the lowest dimensional class one representation $\mu$ with deg $>3$ is the unique 5 dimensional representation of $S O(3)$ which is also faithful. If $m=5$ and $n=7$, the manifold $M$ has dimension 20 and isotropy groups

$$
\mu(S O(2)) \times S O(2) \subset\{\mu(S O(3)) \times S O(2), \mu(S O(2)) \times S O(3)\} \subset S O(7) .
$$

Notice that this example is already covered by Theorem 3.2 in GVWZ.

We now describe the explicit embedding of the groups. $S O(m) \times S O(n-m)$ is embedded in $G=S O(n)$ block-wise, i.e., $S O(m)$ sits in the upper-left $m \times m$-block and $S O(n-m)$ is in the lower-right block. By assumption, $\rho$ is a faithful orthogonal representation of $K^{\prime}$ with representation space $V=\mathbb{R}^{m}$. Let $W_{1}, \ldots, W_{\alpha}$ be invariant subspaces of $V$ such that they are pairwisely orthogonal, and the restrictions of $\left.\rho\left(K^{\prime}\right)\right|_{W_{i}}=\mu$ are equivalent and irreducible. Let $U$ be the orthogonal complement of $W=W_{1} \oplus \cdots \oplus W_{\alpha}$. According to the decomposition of $V$ into invariant spaces, we can write $\rho$ as

$$
\rho=\tau \oplus \mu \oplus \cdots \oplus \mu,
$$

where $\tau$ is the restriction of $\rho$ to $U$ and $\mu$ is the class one representation of $\left(K^{\prime}, H^{\prime}\right)$. Furthermore, $\mu$ is not a subrepresentation of $\tau$. Suppose $r=\operatorname{dim} U, l=\operatorname{dim} W_{i}=$ $\operatorname{deg} \mu$; then $m=r+\alpha l$. By choosing a suitable basis of $V, \rho(x)$ for $x \in K^{\prime}$, is a block diagonal matrix in $S O(m)$ :

$$
\rho(x)=\operatorname{diag}\{\tau(x), \mu(x), \cdots, \mu(x)\} \in S O(r) \times S O(l) \times \cdots \times S O(l) .
$$

When $\mu$ is restricted to the subgroup $H^{\prime} \subset K^{\prime}$, it is not irreducible any more. Let $m_{0}$ be the multiplicity of the trivial representation in the restriction $\left.\mu\right|_{H^{\prime}}$. Hence for any element $y \in H^{\prime}, \mu(y) \in S O\left(l-m_{0}\right) \subset S O(l)$ and $S O\left(l-m_{0}\right)$ is embedded as the upper-left $\left(l-m_{0}\right) \times\left(l-m_{0}\right)$ block in $S O(l)$.

With the explicit description of the embeddings, we have

Proposition 3.9. The Weyl group $\mathcal{W}$ is isomorphic to $\mathbb{Z}_{2} \times \mathbb{Z}_{2}$.

Proof. For any element $x \in K^{\prime}$ and $A \in S O(n-m)$, let $M(x, A)$ denote the block diagonal matrix $\operatorname{diag}(\rho(x), A)$ with $\rho(x) \in S O(m)$. If $x \in H^{\prime}, A$ can be considered as a matrix in $S O(n-m+1)$ since $\rho(x) \in S O(m-1)$.

First notice that $w_{+}$can be represented by the element $a \in K^{+}=\rho\left(H^{\prime}\right) \times$ $S O(n-m+1)$ which is not in $H$, but $a^{2} \in H$. Let

$$
w_{+}=M\left(\mathrm{id},\left(\begin{array}{ccc}
-1 & & \\
& -1 & \\
& & I_{n-m-1}
\end{array}\right)\right)=a=\operatorname{diag}\left\{I_{m-1},-1,-1, I_{n-m-1}\right\},
$$

where $I_{k}$ is the $k \times k$ identity matrix.

Suppose $b=M(x, A)$ is a representative of $w_{-}$, i.e., $b \in N_{K^{-}}(H)$ and $b^{2}$ but not $b \in H$. In the following we will determine the element $b$ in three different cases depending on the class one representation $\mu$. 
Case 1: $\mu$ is of real type and $m_{0}=1$. In each $W=W_{i}(i=1, \ldots, \alpha)$, let $v$ be a unit vector fixed by $\mu\left(H^{\prime}\right)$ (or equivalently by $H$ ) and $X$ be its orthogonal complement. Then $\operatorname{dim} X=l-1$. Since $b \in N_{K^{-}}(H), b . v$ is also fixed by $H$ and has the same length as $v$, i.e., $b . v= \pm v$. Since $b$ is an orthogonal transformation, $b$ maps $X$ to itself, i.e., when restricted on $W, b=\left(\begin{array}{cc}b_{1} & 0 \\ 0 & \operatorname{det} b_{1}\end{array}\right)$ where $b_{1} \in O(l-1)$. Therefore the representative $b$ has the following block diagonal matrix form:

$$
w_{-}=b=\operatorname{diag}\left\{A_{1}, A_{2}, \operatorname{det} A_{2}, \cdots, A_{2}, \operatorname{det} A_{2}, I_{n-m}\right\},
$$

where $A_{1}=\tau(x) \in S O(r), A_{2}=b_{1} \in O(l-1)$ and there are $\alpha$ copies of $\left({ }^{A_{2}} \operatorname{det} A_{2}\right)$.

Case 2: $\mu$ is not of real type and $m_{0}=2$. In this case we have $\mu=\nu \oplus \nu^{*}$, where $\nu$ is a complex class one representation for the pair $\left(K^{\prime}, H^{\prime}\right)$. If $\nu$ is of quaternionic type, then $\nu^{*}=\nu$ and $\mu=\nu \oplus \nu$. As in Case 1 , we have $W=X \oplus_{\perp} Y$, where $Y$ is the 2 dimensional subspace fixed by $\mu\left(H^{\prime}\right)$ and $X$ is its orthogonal complement. The orthogonal transformation $\mu(x)$ maps $X$ and $Y$ to themselves and the matrix $\mu(x)$ has the form $\left(\begin{array}{cc}b_{1} & 0 \\ 0 & b_{2}\end{array}\right) \in S(O(l-2) \times O(2))$. Since $\mu(x)^{2} \in H$ implies $b_{2}^{2}=I_{2}$, $b_{2}$ is a symmetric matrix. Since $\mu=\nu \oplus \nu^{*}, b_{2}$ commutes with the matrix $\left(\begin{array}{cc}0 & -1 \\ 1 & 0\end{array}\right)$ which implies $b_{2}= \pm I_{2}$. Therefore $b$ also has the matrix form as in (3.11).

Case 3: $\mu$ is not in Case 1 or Case 2. From the classification of class one representations in Theorem A.2 and their types in Proposition A.3, the only representation of this type is $\left(K^{\prime}, H^{\prime}\right)=(S p(k), S p(k-1))$, where $\mu$ has the highest weight $p \varpi_{1}+q \varpi_{2}$ with $p \geq 1$. Here $\varpi_{1}$ and $\varpi_{2}$ are the 1 st and 2 nd fundamental weights of $S p(n)$. Since $\rho$ is a faithful representation, we have $x \in K^{\prime} \cap N_{K^{\prime}}\left(H^{\prime}\right)-H^{\prime}$ and $x^{2} \in H^{\prime}$. Take $x=-I_{k} \in S p(k)$; then $x$ satisfies these restrictions. If we view $S p(k)$ as a subgroup of $S O(4 k)$, then $\mu$ is contained in the restriction of some class one representation $\nu$ of $S O(4 k)$ to $S p(k)$ by Theorem A.1. The representation space of $\nu$ consists of homogeneous harmonic polynomials, so the image $\nu(x)$ is $\pm \operatorname{Id}$ depending on the parity of the degree of the polynomials. Therefore $\mu(x)$ is equal to $\pm \operatorname{Id}$ and the element $w_{-}$can be represented by the following block diagonal matrix:

$$
w_{-}=b=\operatorname{diag}\left\{A_{1}, \varepsilon I_{\alpha l}, I_{n-m}\right\},
$$

where $A_{1}=\tau(x) \in S O(r)$ and $\mu(x)=\varepsilon I_{l}$ with $\varepsilon= \pm 1$.

In each case, from the given representatives of $w_{ \pm}$, it is easy to check that $w_{+} w_{-}=w_{-} w_{+}$, which is not an element in $H$. Thus $\mathcal{W}=\left\langle w_{-}, w_{+}\right\rangle \cong \mathbb{Z}_{2} \times \mathbb{Z}_{2}$.

Remark 3.13. Let $a$ be an element in $N(H)_{0}$ which does not lie in $N\left(K^{-}\right)$or $N\left(K^{+}\right)$and let $\bar{M}$ be the cohomogeneity one manifold defined by the group diagram $H \subset\left\{K^{-}, a K^{+} a^{-1}\right\} \subset G$. As pointed out in Remark 2.3, $M$ and $\bar{M}$ usually have different Weyl groups and different invariant metrics though they are $G$-equivariantly diffeomorphic. Therefore, if one family of invariant metrics does not admit non-negative curvature, it does not necessarily follow that the other family is obstructed as well. In our example, if the multiplicity of the trivial representation in $\left.\mu\right|_{H^{\prime}}$ is equal to one, i.e., $m_{0}=1$, then our arguments which show the obstructions work for all equivalent diagrams. This is the case for most of the class one representations of spherical pairs; see, for example, Theorem A.2. On the other hand, if $m_{0} \geq 2$, then we have to put further restriction on the diagram: the Lie algebra of $K^{+}$contains the subspace $\operatorname{span}\left\{E_{m, m+1}, \ldots, E_{m, n}\right\}$. Here we use 
$E_{i, j}, i \neq j$, to denote the skew-symmetric matrix having 1 in the $i, j$-entry, -1 in the $j, i$-entry and zero otherwise.

Using the explicit representatives of the generators of the Weyl group, we have the following smoothness condition of an invariant metric on $M$.

Lemma 3.14. For any $G$ invariant metric $g$ on $M$, let $h(t)$ be the length of the Killing vector field generated by $E_{m, m+1}$ along the geodesic $c(t)$. Then $h(t)$ is an even function with $h(0) \neq 0$ and $h(L)=0$.

Proof. The fact that $E_{m, m+1}$ lies in the Lie algebra of $K^{+}$but not $K^{-}$implies that $h(0) \neq 0$ and $h(L)=0$. The generator $w_{-}$is a reflection of $c(t)$ at the point $p_{-}$and maps $c(t)$ to $c(-t)$. The induced map $\mathrm{d} w_{-}$takes $T_{c(t)} M$ to the tangent space $T_{c(-t)} M$. From the matrix form (3.11) and (3.12) of a representative of $w_{-}$, we have $\mathrm{d} w_{-}\left(E_{m, m+1}^{*}(t)\right)= \pm E_{m, m+1}^{*}(-t)$. Therefore $h(t)=h(-t)$, i.e., $h(t)$ is an even function.

\section{Restrictions on the Metric ALONG the nORMAL GeOdesic}

In this section, we begin the study of the invariant metrics in our examples. We recall the rigidity results by Wilking.

Let $c: \mathbb{R} \longrightarrow M$ be a geodesic and let $\Lambda$ be an $(n-1)$ dimensional family of normal Jacobi fields. The Ricatti operator $L(t)$ is the endomorphism of the normal bundle $(\dot{c}(t))^{\perp}$ defined by $L(t) J(t)=J^{\prime}(t)$ for $J \in \Lambda$. From Theorem 9 and Corollary 10 in $[\mathrm{Wi}$, we have

Theorem 4.1 (Wilking's Rigidity Theorem). Suppose the Riccati operator for an $(n-1)$ dimensional family $\Lambda$ of normal Jacobi fields is self-adjoint, and define the smooth subbundle $\Upsilon$ of $(\dot{c}(t))^{\perp}$ by

$$
\Upsilon=\operatorname{span}\{J \in \Lambda \mid J(t)=0 \text { for some } t \in \mathbb{R}\} .
$$

Then, if $M$ has non-negative curvature, we have:

$$
\Lambda=\Upsilon \oplus\{J \in \Lambda \mid J \text { is parallel }\}
$$

and

$$
\Upsilon(t)=\{J(t) \mid J \in \Upsilon\} \oplus\left\{J^{\prime}(t) \mid J \in \Upsilon, J(t)=0\right\} .
$$

A point $t_{0} \in \mathbb{R}$ or $c\left(t_{0}\right)$ is said to be singular if $J\left(t_{0}\right)=0$ for some $J \in \Upsilon$. Otherwise $t_{0}$ is said to be generic. Thus if $J \in \Lambda$ and $J\left(t_{0}\right) \perp \Upsilon\left(t_{0}\right)$ at a generic $t_{0}$, then $J$ is parallel along $c(t), t \in \mathbb{R}$.

Remark 4.3. If there exists a subbundle $E \subset(\dot{c}(t))^{\perp}$ that is invariant under parallel transport, then Theorem 4.1 can also be applied to a rank $E$ dimensional family of normal Jacobi fields in $E$ with self-adjoint Ricatti operator.

In our example, let $c(t)$ be the minimal geodesic between $B_{ \pm}$, and let $X^{*}(c(t))$, $X \in \mathfrak{h}^{\perp}$, an $(n-1)$ dimensional family of Jacobi fields. Its Riccati operator $L(t)$ is self-adjoint, since it is equal to the shape operator $-\frac{1}{2} P_{t}^{-1} P_{t}^{\prime}$; see equation (2.6). We will apply Theorem 4.1 to a subfamily of these Jacobi fields.

Let $\mathfrak{g}=\mathfrak{s o}(n)$ and $\mathfrak{h}$ be the Lie algebras of $G=S O(n)$ and $H=\rho\left(K^{\prime}\right) \times S O(n-m)$ respectively. Choose the bi-invariant inner product $Q=-\frac{1}{2} \operatorname{Tr}$ on $\mathfrak{g}$ for which $\left\{E_{i, j}\right\}$ is an orthonormal basis and let $\mathfrak{p}$ be the orthogonal complement of $\mathfrak{h} \subset \mathfrak{g}$. First we identify some subspaces of $\mathfrak{p}$. 
Let

$$
\begin{aligned}
\mathfrak{q}_{0} & =\operatorname{span}\left\{E_{i, j} \mid 1 \leq i \leq r, m+1 \leq j \leq n\right\}, \\
\mathfrak{q}_{1} & =\operatorname{span}\left\{E_{i, j} \mid r+1 \leq i \leq r+l, m+1 \leq j \leq n\right\} \\
& \ldots \\
\mathfrak{q}_{\alpha} & =\operatorname{span}\left\{E_{i, j} \mid m+1-l \leq i \leq m, m+1 \leq j \leq n\right\},
\end{aligned}
$$

and $\mathfrak{q}=\mathfrak{q}_{0}+\mathfrak{q}_{1}+\cdots+\mathfrak{q}_{\alpha}$. We write the last subspace $\mathfrak{q}_{\alpha}$ as a sum of two subspaces as follows:

$$
\begin{aligned}
& \mathfrak{n}_{1}=\operatorname{span}\left\{E_{i, j} \mid m+1-l \leq i \leq m-1, m+1 \leq j \leq n\right\}, \\
& \mathfrak{n}_{2}=\operatorname{span}\left\{E_{m, j} \mid m+1 \leq j \leq n\right\} .
\end{aligned}
$$

Let $\mathfrak{q}^{\perp}$ be the $Q$-orthogonal complement of $\mathfrak{q}$ in $\mathfrak{p}$. Since $\mathfrak{q}^{\perp}$ is the fixed point set by the isotropy action of the subgroup $S O(n-m) \subset H=\rho\left(H^{\prime}\right) \times S O(n-m)$, Schur's lemma implies that the Killing vector field $X^{*}, X \in \mathfrak{q}^{\perp}$, is orthogonal along $c(t)$ to $Y^{*}$ for any $Y \in \mathfrak{q}$.

Terminology. In the rest of the paper, for any two subspaces $\mathfrak{p}_{1}, \mathfrak{p}_{2} \subset \mathfrak{p}$, the notation $\mathfrak{p}_{1}^{*} \perp \mathfrak{p}_{2}^{*}$ means that any Killing vector field generated by an element in $\mathfrak{p}_{1}$ is orthogonal to any Killing vector field generated by an element in $\mathfrak{p}_{2}$ along $c(t)$.

Since parallel translation commutes with the action of $\operatorname{Ad}_{H}$, and since $\mathfrak{q}^{\perp}$ is the fixed point set of $S O(n-m)$ in $H$, it follows that $\left(\mathfrak{q}^{\perp}\right)^{*}$, and hence also $\mathfrak{q}^{*}$, is invariant under parallel translation. By the same reasoning, $P^{-1} P^{\prime}$ preserves $\mathfrak{q}^{*}$, and thus $\mathfrak{q}^{*}$ forms a self-adjoint family of Jacobi fields to which we can thus apply Theorem 4.1 .

We determine the component $\Upsilon$ in the splitting (4.2) of the Jacobi fields $\mathfrak{q}^{*} \cdot p_{+}=$ $c(L)$ is a singular point. The element $w_{+}$fixes $p_{+}$and reflects $c(t)$ about $p_{+}$. Let $q_{-}=c(2 L)=w_{+}\left(p_{-}\right) \in B_{-}$; then the isotropy subgroup at $q_{-}$is $K_{1}^{-}=\operatorname{Ad}_{w_{+}} K^{-}$ with Lie algebra $\mathfrak{k}_{1}^{-}=\operatorname{Ad}_{w_{+}} \mathfrak{k}^{-}$. Similarly, $w_{-}$fixes $p_{-}$and reflects $c(t)$ about $p_{-}$. Let $q_{+}=c(-L)=w_{-}\left(p_{+}\right) \in B_{+}$; then $q_{+}$has isotropy subgroup $K_{1}^{+}=\operatorname{Ad}_{w_{-}} K^{+}$ with Lie algebra $\mathfrak{k}_{1}^{+}=\operatorname{Ad}_{w_{-}} \mathfrak{k}^{+}$. Since $w_{-} . w_{+}=w_{+} . w_{-}$, the image of $q_{-}$under the reflection $w_{-}$about $p_{-}$is $w_{-}\left(q_{-}\right)=w_{-} . w_{+}\left(p_{-}\right)=w_{+} . w_{-}\left(p_{-}\right)=w_{+}\left(p_{-}\right)=q_{-}$, i.e., $c(2 L)=c(-2 L)$. Therefore $c(t)$ is a closed geodesic with period $4 L$ and the singular points are $p_{+}=c(L)$ and $q_{+}=c(3 L)$. The vanishing Killing vector fields are those generated by the vectors in the Lie algebras of the isotropy subgroups at singular points. Notice that if $X \in \mathfrak{q}$, then $X^{*}\left(p_{-}\right) \neq 0$ and $X^{*}\left(q_{-}\right) \neq 0$. Theorem 4.1 implies

Lemma 4.6. If $Y \in \mathfrak{q}$ such that $Y^{*}\left(p_{-}\right) \perp X^{*}\left(p_{-}\right)$for all $X \in \mathfrak{n}_{2}$, then $Y^{*}$ is a parallel Jacobi field along $c(t)$.

In the following, we prove some properties of the invariant metrics $g$ on $M$ under the non-negative sectional curvatures assumption.

First we observe

Lemma 4.7. Suppose $(M, g)$ is non-negatively curved; then $\mathfrak{q}_{0}^{*}$ is orthogonal to $\left(\mathfrak{q}_{1}+\cdots+\mathfrak{q}_{\alpha}\right)^{*}$ along $c(t)$.

Proof. At the generic point $p_{-}=c(0)$, the metric $g$ restricted to the singular orbit $B_{-} \cong G / K^{-}$is $\operatorname{Ad}_{K^{-}}$invariant. The actions of $\operatorname{Ad}_{K^{-}}$on $\mathfrak{q}_{0}$ and $\mathfrak{q}_{i}(i>0)$ are $\tau \otimes \rho_{n-m}$ and $\mu \otimes \rho_{n-m}$ respectively, where $\varrho_{n-m}$ is the standard representation 
of $S O(n-m)$ on $\mathbb{R}^{n-m}$. Since $\tau$ does not contain $\mu$ as a subrepresentation, $\mathfrak{q}_{0}^{*}$ is orthogonal to $\mathfrak{q}_{i}^{*}$ at $p_{-}$. In particular $\mathfrak{q}_{0}^{*}$ is orthogonal to $\mathfrak{n}_{2}^{*}$, so any Killing vector field generated by a vector in $\mathfrak{q}_{0}$ is parallel along $c(t)$. Hence $\mathfrak{q}_{0}^{*}$ is orthogonal to $\left(\mathfrak{q}_{1}+\cdots+\mathfrak{q}_{\alpha}\right)^{*}$ along $c(t)$.

Next we study the metric $g$ on the space $\left(\mathfrak{q}_{1}+\cdots+\mathfrak{q}_{\alpha}\right)^{*}$. Recall $h^{2}(t)=$ $g\left(E_{m, m+1}^{*}, E_{m, m+1}^{*}\right)$ is an even function with $h(0) \neq 0$ and w.l.o.g. we may assume that $h(0)=1$.

4.1. $\mu$ is of real type or the multiplicity of $\mu$ in $\rho$ is one. We first consider the case where $\mu$ is of real type. We denote $E_{r+(i-1) l+a, m+\xi}$ by $E_{a, i, \xi}$ for $a=1, \ldots, l$, $i=1, \ldots, \alpha$ and $\xi=1, \ldots, n-m$.

Since $\operatorname{Ad}_{K^{-}}$commutes with $P(0)$, the restriction of $P(0)$ to $\mathfrak{q}_{i}$, composed with the projection to $\mathfrak{q}_{j}$, is an equivalence between the $K^{-}$irreducible representations $\mathfrak{q}_{i}$ and $\mathfrak{q}_{j}$. Since they are orthogonal, Schur's lemma implies that $P(0)\left(E_{a, i, \xi}\right)=$ $f_{i, j} E_{a, j, \xi}$ for some constant $f_{i, j} \in \mathbb{R}$. Furthermore, $f_{i, j}=f_{j, i}$ from the $Q$-symmetry of $P_{t}$. In terms of an inner product of Killing vector fields, we have

$$
f_{i, j}=g\left(E_{1, i, 1}^{*}, E_{1, j, 1}^{*}\right)_{c(0)} \text {. }
$$

The assumption $h(0)=1$ implies that $f_{\alpha, \alpha}=1$.

Lemma 4.9. Suppose $(M, g)$ is non-negatively curved. Then we have

(1) $E_{a, i, \xi}^{*}$ is a parallel Jacobi field along $c(t)$ if $a \neq l$;

(2) $E_{a, i, \xi}^{*}$ is orthogonal to $E_{b, j, \zeta}^{*}$ along $c(t)$ if $a \neq b$ or $\xi \neq \zeta$;

(3) $E_{a, i, \xi}^{*}$ has the same length as $E_{a, i, \zeta}^{*}$ along $c(t)$;

(4) at the point $p_{-}=c(0), P_{0}\left(E_{a, i, \xi}\right)=\sum_{j=1}^{\alpha} E_{a, j, \xi} f_{i, j}$.

Proof. At the generic point $c(0)$, the $\operatorname{Ad}_{K^{-}}$actions on $\mathfrak{q}_{i}$ and $\mathfrak{q}_{j}$ are equivalent and given by the irreducible representation $\mu \otimes \varrho_{n-m}$, from Schur's lemma and the fact that $\mu$ is of real type or the multiplicity of $\mu$ in $\rho$ is one, $E_{a, i, \xi}^{*}(0)$ is orthogonal to $\Upsilon(0)=\operatorname{span}\left\{E_{l, \alpha, \varsigma}^{*}(0) \mid \varsigma=1, \ldots, n-m\right\}$ for $a \neq l$. Hence it is a parallel vector field from the last part of Theorem 4.1, which proves (1).

On each principal orbit $M_{t} \cong G / H, \operatorname{Ad}_{H}$ acts on each $\mathfrak{q}_{i}(i>0)$ by the representation $\operatorname{Res}_{H^{\prime}}^{K^{\prime}}(\mu) \otimes \varrho_{n-m}$. By Schur's lemma we have that $E_{a, i, \xi}^{*}$ is orthogonal to $E_{b, j, \zeta}^{*}$ along $c(t)$ if $\xi \neq \zeta$ and $E_{a, i, \xi}^{*}$ has the same length as $E_{a, i, \zeta}^{*}$. This proves (3) and one case of $(2)$ where $\xi \neq \zeta$.

Suppose $\zeta=\xi$ and $a \neq b$. If none of $a$ or $b$ is equal to $l$, then the two vector fields $E_{a, i, \xi}^{*}$ and $E_{b, j, \xi}^{*}$ are parallel from (1). Using Schur's lemma again and the fact that $a \neq b$, they are orthogonal to each other at $c(0)$ and then along the normal geodesic $c(t)$.

If one of $a$ and $b$, say $b$, is equal to $l$, then $E_{a, i, \xi}^{*}$ is a parallel vector field. Write $E_{l, j, \xi}^{*}(0)=\left(E_{l, j, \xi}-\lambda E_{l, \alpha, \xi}\right)^{*}(0)+\lambda E_{l, \alpha, \xi}^{*}(0)$, where the constant $\lambda$ is determined by the following equation:

$$
g\left(E_{l, j, \xi}^{*}, E_{l, \alpha, \xi}^{*}\right)_{c(0)}=\lambda g\left(E_{l, \alpha, \xi}^{*}, E_{l, \alpha, \xi}^{*}\right)_{c(0)} .
$$

Thus $\left(E_{l, j, \xi}-\lambda E_{l, \alpha, \xi}\right)^{*}(0) \perp \Upsilon(0)$ and hence $\left(E_{l, j, \xi}-\lambda E_{l, \alpha, \xi}\right)^{*}$ is a parallel vector field. Furthermore, $E_{a, i, \xi}^{*}$ is orthogonal to $\left(E_{l, j, \xi}-\lambda E_{l, \alpha, \xi}\right)^{*}$ at $c(0)$, so they are orthogonal to each other along $c(t)$. Thus $E_{a, i, \xi}^{*}$ is orthogonal to $E_{l, j, \xi}^{*}$ along $c(t)$.

The formula of $P_{0}\left(E_{a, i, \xi}\right)$ in (4) follows easily from the defining equation (4.8) of $f_{i, j}$ and (2). 
From Lemma 4.9 above, the restriction of the endomorphism $P$ on $\left(\mathfrak{q}_{1}+\cdots+\mathfrak{q}_{\alpha}\right)^{*}$ at $t=0$ has the following matrix form:

$$
P_{0}=\left(\begin{array}{ccc}
f_{1,1} I_{l} & \cdots & f_{1, \alpha} I_{l} \\
\vdots & \ddots & \vdots \\
f_{\alpha, 1} I_{l} & \cdots & f_{\alpha, \alpha} I_{l}
\end{array}\right) .
$$

We have seen that there are plenty of parallel Killing vector fields in $\left(\mathfrak{q}_{1}+\cdots+\right.$ $\left.\mathfrak{q}_{\alpha}\right)^{*}$. Using these parallel vector fields, we can determine the restriction of $P_{t}$ on $\left(\mathfrak{q}_{1}+\cdots+\mathfrak{q}_{\alpha}\right)^{*}$.

Theorem 4.11. If the cohomogeneity one manifold $(M, g)$ has non-negative sectional curvature and the class one representation $\mu$ is of real type, then for any $i=1, \ldots, \alpha$ and $\xi=1, \ldots, n-m$, we have

(1) $P_{t}\left(E_{a, i, \xi}\right)=\sum_{j=1}^{\alpha} f_{i, j} E_{a, j, \xi}$, for $a=1, \cdots, l-1$;

(2) $P_{t}\left(E_{l, i, \xi}\right)=\sum_{j=1}^{\alpha} p_{i, j}(t) E_{l, j, \xi}$ and $p_{i, j}(t)$ is defined as

$$
p_{i, j}(t)=\left(h^{2}(t)-1\right) a_{i} a_{j}+f_{i, j},
$$

where $a_{i}=f_{i, \alpha}$ and $a_{\alpha}=1$.

Proof. Part (1) is obvious since every component of both $E_{a, i, \xi}^{*}$ and $E_{a, j, \xi}^{*}$ are parallel vector fields along $c(t)$ if $a \leq l-1$.

For part (2), let

$$
X_{i}=E_{l, i, \xi}-a_{i} E_{l, \alpha, \xi}, \quad i=1, \ldots, \alpha .
$$

Then $X_{i} \in \mathfrak{q}_{1}+\cdots+\mathfrak{q}_{\alpha}$ and generates a Killing vector field $X_{i}^{*}$ along $c(t)$. By the definition $a_{i}=f_{i, \alpha}$, the defining equation of $f_{i, \alpha}$ in (4.8) and (4) in Lemma 4.9, we have

$$
g\left(X_{i}^{*}, E_{l, \alpha, \xi}^{*}\right)_{c(0)}=0,
$$

or $X_{i}^{*}(0) \perp \Upsilon(0)$. Therefore $X_{i}^{*}$ is a parallel vector field. By formula (2.6) of the shape operator, we have

$$
P_{t}^{\prime}\left(X_{i}\right)=0 \quad \forall t \in \mathbb{R} .
$$

Since $P_{t}\left(E_{l, i, \xi}\right)=\sum_{j=1}^{\alpha} p_{i, j}(t) E_{l, j, \xi}$ for some functions $p_{i, j}(t)$, we have

$$
\begin{aligned}
& P_{t}^{\prime}\left(E_{l, i, \xi}-a_{i} E_{l, \alpha, \xi}\right)=P_{t}^{\prime}\left(E_{l, i, \xi}\right)-a_{i} P_{t}^{\prime}\left(E_{l, \alpha, \xi}\right) \\
= & \sum_{j=1}^{\alpha} p_{i, j}^{\prime}(t) E_{l, j, \xi}-a_{i} \sum_{j=1}^{\alpha} p_{\alpha, j}^{\prime}(t) E_{l, j, \xi}=0 .
\end{aligned}
$$

Therefore we have the following system of ordinary differential equations for $p_{i, j}(t)$ :

$$
p_{i, j}^{\prime}(t)-a_{i} p_{\alpha, j}^{\prime}(t)=0 . \quad \forall i, j=1, \ldots, \alpha .
$$

One easily sees that it has the solution

$$
p_{i, j}(t)=a_{i} a_{j} h^{2}(t)+f_{i, j}-a_{i} f_{j, \alpha},
$$

which finishes our proof.

Now we consider the case when the multiplicity of $\mu$ in $\rho$ is one, i.e., $\alpha=1$. Since $P_{1,1}(0)$ is symmetric and $\operatorname{Ad}_{K^{-}}$equivariant, the off-diagonal terms in formulas (2.9) and (2.10) of equivariant endomorphisms vanish, i.e., $P_{1,1}(0)=f_{1,1} I_{l}$ with some constant $f_{1,1} \in \mathbb{R}$. From a similar argument as in the previous case, we have 
Theorem 4.14. If the cohomogeneity one manifold $(M, g)$ has non-negative sectional curvature and the multiplicity of the class one representation $\mu$ in $\rho$ is one, then for any $\xi=1, \ldots, n-m$, we have

(1) $P_{t}\left(E_{a, 1, \xi}\right)=f_{1,1} E_{a, 1, \xi}$, if $a=1, \cdots, l-1$;

(2) $P_{t}\left(E_{l, 1, \xi}\right)=p_{1,1}(t) E_{l, 1, \xi}$ and $p_{1,1}(t)=\left(h^{2}(t)-1\right) f_{1,1}^{2}+f_{1,1}$.

4.2. $\mu$ is of complex or quaternionic type. We consider the complex case first and the quaternionic case will follow easily.

Let $l=2 p$ and $\beta=2 \alpha$. Recall that $W_{i}$ is the subspace of $V$ such that $\left.\rho\right|_{W_{i}}=\mu$. Choose an orthonormal basis $\left\{e_{1}, \ldots, e_{2 p}\right\}$ of $W_{i}$ such that $\mu(x)$ has the form $\left(\begin{array}{cc}A & -B \\ B & A\end{array}\right)$ with $A, B$ being $p \times p$ matrices and $\mu\left(H^{\prime}\right)$ fixes the two vectors $e_{p}$ and $e_{2 p}$. Under this basis any $\operatorname{Ad}_{\mu\left(K^{\prime}\right)}$-equivariant endomorphism has the block-form $\left(\begin{array}{cc}a I_{p} & -b I_{p} \\ b I_{p} & a I_{p}\end{array}\right)$ as in (2.9) with constants $a, b \in \mathbb{R}$. Using the fact that the endomorphism commutes with the rotation in the $\left\{e_{p}, e_{2 p}\right\}$ plane, we may assume that $E_{m, m} \in K^{+}$.

Since $\mu$ is of complex type, the $\operatorname{Ad}_{K^{-}}$-equivariant map $P(0)$ has a block form and the $(i, j)$-block is given by

$$
\left(\begin{array}{cc}
f_{2 i-1,2 j-1} I_{p} & f_{2 i, 2 j-1} I_{p} \\
f_{2 i-1,2 j} I_{p} & f_{2 i, 2 j} I_{p}
\end{array}\right)
$$

where $f_{a, b} \in \mathbb{R}$ is constant for $a, b=1, \ldots, \beta=2 \alpha$ and satisfies the following identities:

$$
\begin{aligned}
& f_{2 i-1,2 j-1}=f_{2 i, 2 j}, \quad f_{2 i, 2 j-1}+f_{2 i-1,2 j}=0, \\
& f_{2 i-1,2 j-1}=f_{2 j-1,2 i-1}, \quad f_{2 i, 2 j-1}+f_{2 j, 2 i-1}=0 .
\end{aligned}
$$

The last two are due to the fact that $P(0)$ is $Q$-symmetric.

Similar to the case when $\mu$ is of real type, we define $E_{a, i, \xi}=E_{r+(i-1) p+a, m+\xi}$ for $a=1, \ldots, p, i=1, \ldots, \beta$ and $\xi=1, \ldots, n-m$. Then we have

Theorem 4.16. If the cohomogeneity one manifold $(M, g)$ has non-negative sectional curvature and the class one representation $\mu$ is of complex type, then for any $i=1, \ldots, \beta$ and $\xi=1, \ldots, n-m$, we have

(1) $P_{t}\left(E_{a, i, \xi}\right)=\sum_{j=1}^{\beta} f_{i, j} E_{a, j, \xi}$, if $a=1, \cdots, p-1$;

(2) $P_{t}\left(E_{p, i, \xi}\right)=\sum_{j=1}^{\beta} p_{i, j}(t) E_{p, j, \xi}$ and $p_{i, j}(t)$ is defined as

$$
p_{i, j}(t)=\left(h^{2}(t)-1\right) a_{i} a_{j}+f_{i, j}
$$

where $a_{i}=f_{i, \beta}$.

Now we consider the case when $\mu$ is of quaternionic type and the multiplicity of $\mu$ in $\rho$ is bigger than one. Let $l=4 p$ and $\beta=4 \alpha$.

From formula (2.10) of equivariant endomorphisms in this case and a similar argument in the complex case, $P(0)=\left(f_{a, b} I_{p}\right)_{1 \leq a, b \leq \beta}$, where $f_{a, b} \in \mathbb{R}$ is constant and satisfies the following identities:

$$
\begin{aligned}
& f_{4 i-3,4 j-3}=f_{4 i-2,4 j-2}=f_{4 i-1,4 j-1}=f_{4 i, 4 j}=f_{4 j, 4 i} \\
& -f_{4 i-2,4 j-3}=f_{4 i-3,4 j-2}=f_{4 i, 4 j-3}=-f_{4 i-1,4 j}=f_{4 j-1,4 i} \\
& -f_{4 i-1,4 j-3}=-f_{4 i, 4 j-2}=f_{4 i-3,4 j-1}=f_{4 i-2,4 j}=-f_{4 j-2,4 i} \\
& -f_{4 i, 4 j-3}=f_{4 i-1,4 j-2}=-f_{4 i-2,4 j-1}=f_{4 i-3,4 j}=-f_{4 j-3,4 i}
\end{aligned}
$$

for $i, j=1, \ldots, \alpha$. 
We denote $E_{r+(i-1) p+a, m+\xi}$ by $E_{a, i, \xi}$ for $a=1, \ldots, p, i=1, \ldots, \beta$ and $\xi=$ $1, \ldots, n-m$, and then we have

Theorem 4.19. If the cohomogeneity one manifold $(M, g)$ has non-negative sectional curvature and the class one representation $\mu$ is of quaternionic type, then for any $i=1, \ldots, \beta$ and $\xi=1, \ldots, n-m$, we have

(1) $P_{t}\left(E_{a, i, \xi}\right)=\sum_{j=1}^{\beta} f_{i, j} E_{a, j, \xi}$, if $a=1, \cdots, p-1$;

(2) $P_{t}\left(E_{p, i, \xi}\right)=\sum_{j=1}^{\beta} p_{i, j}(t) E_{p, j, \xi}$ and $p_{i, j}(t)$ is defined as

$$
p_{i, j}(t)=\left(h^{2}(t)-1\right) a_{i} a_{j}+f_{i, j},
$$

where $a_{i}=f_{i, \beta}$.

\section{Proof of Theorem 3.1}

In this section, we develop a contradiction from the assumption that the manifold $(M, g)$ is non-negatively curved, which finishes the proof of Theorem 3.1

Lemma 5.1. The image $\mu\left(K^{\prime}\right) \subset S O(l)$ does not act transitively on the sphere $\mathbb{S}^{l-1}=S O(l) / S O(l-1)$.

Proof. Recall that $l=\operatorname{deg} \mu \geq k+2$. If $\mu\left(K^{\prime}\right)$ could act transitively on $\mathbb{S}^{l-1}$, then $K^{\prime}$ would act transitively on both $\mathbb{S}^{k}$ and $\mathbb{S}^{l-1}$ and $l-1 \geq k+1$. By the classification of the transitive action on the spheres, we have that $\left(K^{\prime}, H^{\prime}\right)$ is either $(S O(7), S O(6))$ with $\mu(S O(7))=S \operatorname{pin}(7) \subset S O(8)$ or $(S O(9), S O(8))$ with $\mu(S O(9))=\operatorname{Spin}(9) \subset$ $S O(16)$. But in both cases, by the classification in Theorem A.2, $\mu$ is not a class one representation for the pair $\left(K^{\prime}, H^{\prime}\right)$, which is a contradiction.

The following was already used in GVWZ and we state it as a lemma without proof.

Lemma 5.2. Suppose $f(t)$ is a $C^{2}$ non-constant even function on $(-\varepsilon, \varepsilon)$ for some $\varepsilon>0$ with $f(0)=0$. Then there is no such constant $\gamma \geq 0$ that satisfies the following inequality:

$$
\gamma^{2}(f(t))^{2}-\left(f^{\prime}(t)\right)^{2} \geq 0 .
$$

From Lemma 3.14 $h(t)$ is an even function with $h(0) \neq 0$ and $h(L)=0$, so $f(t)=h^{2}(0)-h^{2}(t)=1-h^{2}(t)$ satisfies the assumptions in Lemma 5.2. We will show that inequality (5.3) holds for some constant $\gamma \geq 0$ using non-negativity of the sectional curvature of a carefully chosen 2-plane. Theorem 3.1 then follows from Lemma 5.2. In the following we use the formula of sectional curvatures in terms of $P_{t}$ given in GZ2, Corollary 1.10].

Proof of Theorem 3.1. In the following argument, only the entries in the lower-right $(n-r) \times(n-r)$-block of $\mathfrak{g}=\mathfrak{s o}(n)$ are involved, so without loss of generality we may assume that $r=0$ or equivalently the representation $\rho$ is a sum of $\alpha$ copies of $\mu$.

Case 1: The representation $\mu$ is of real type. Since $P_{0}$ defined in (4.10) is symmetric and positive definite, we can write

$$
\left(f_{i j}\right)_{1 \leq i, j \leq \alpha}=A D A^{\top}
$$


where $A=\left(A_{i, j}\right)_{\alpha \times \alpha}$ is an orthogonal matrix and $D=\operatorname{diag}\left(d_{1}, \ldots, d_{\alpha}\right)$ is a diagonal matrix with positive entries. Define the following vectors in $\mathfrak{q}_{1}+\cdots+\mathfrak{q}_{\alpha}$ :

$$
\begin{aligned}
X^{u} & =\sum_{i=1}^{l-1} b_{i} E_{i, u, 1}+E_{l, u, 2}, \\
Y^{u} & =\sum_{i=1}^{l-1} b_{i} E_{i, u, 2}+E_{l, u, 1},
\end{aligned}
$$

where $u=1, \ldots, \alpha$ and $\sum_{i=1}^{l-1} b_{i}^{2}=1$. Further conditions of the $b_{i}$ 's will be determined later on.

In the matrix $A$, there is a column, say the $i_{0}$-th column, with $A_{\alpha, i_{0}} \neq 0$. We denote $A_{u, i_{0}}$ by $A_{u}, u=1, \ldots, \alpha$, and define the following two vectors $X, Y$ in $\mathfrak{p}$ :

$$
X=\sum_{u=1}^{\alpha} A_{u} X^{u}, \quad Y=\sum_{u=1}^{\alpha} A_{u} Y^{u} .
$$

From the definitions of $X^{u}$ and $Y^{v}$, it is easy to see that $\left[X^{u}, Y^{u}\right]=0$, and if $u \neq v$, then

$$
\left[X^{u}, Y^{v}\right]=\sum_{i=1}^{l-1} b_{i}\left(E_{v l, i+(u-1) l}+E_{i+(v-1) l, u l}\right) .
$$

Hence

$$
\begin{aligned}
{[X, Y] } & =\sum_{u, v=1}^{\alpha} A_{u} A_{v}\left[X^{u}, Y^{v}\right]=\sum_{i=1}^{l-1} \sum_{u \neq v} A_{u} A_{v} b_{i}\left(E_{v l, i+(u-1) l}+E_{i+(v-1) l, u l}\right) \\
& =0 .
\end{aligned}
$$

The fact that $X$ commutes with $Y$ makes the computation of the sectional curvature of the 2-plane spanned by $X^{*}$ and $Y^{*}$ easier since the first two terms in the curvature formula $(a)$ in [GZ2, Corollary 1.10] vanish. The other four terms are computed in Proposition 5.7 below. If we plug the result of each term into the formula of the sectional curvature, we have

$$
\begin{aligned}
\left\|X^{*} \wedge Y^{*}\right\|^{2} \sec \left(X^{*}, Y^{*}\right)_{c(t)} & =\left(h^{2}(t)-1\right)^{2} Q\left(X_{0}, P_{t}^{-1}\left(X_{0}\right)\right)-\left(d_{i_{0}} A_{\alpha}\right)^{4} h^{2}(t)\left(h^{\prime}(t)\right)^{2} \\
& -Q\left(A_{+}(X, X), P_{t}^{-1} A_{+}(X, X)\right) .
\end{aligned}
$$

Here $X_{0} \in \mathfrak{p}$ is specified in Proposition 5.7 and is orthogonal to $\mathfrak{k}^{-}$with respect to $Q$.

Since $P_{t}$ is positive definite as well as $P_{t}^{-1}$, we have $Q\left(A_{+}(X, X), P_{t}^{-1} A_{+}(X, X)\right)$ $\geq 0$. Therefore $\sec \left(X^{*}, Y^{*}\right) \geq 0$ implies that

$$
\left(h^{2}(t)-1\right)^{2} Q\left(X_{0}, P_{t}^{-1}\left(X_{0}\right)\right)-\left(d_{i_{0}} A_{\alpha}\right)^{4} h^{2}(t)\left(h^{\prime}(t)\right)^{2} \geq 0 .
$$

The existence of the constant $\gamma \geq 0$ follows from the fact that $A_{\alpha} \neq 0$ and $Q\left(X_{0}, P_{t}^{-1}\left(X_{0}\right)\right)$ is bounded from above near $t=0$.

Case 2: The representation $\mu$ is not of real type and the multiplicity of $\mu$ in $\rho$ equals 1. In this case $P_{0}$ is a scalar matrix and $P_{t}$ is a diagonal matrix. It is easy to see that the proof in the previous case works if we choose $X=X^{1}$ and $Y=Y^{1}$ in (5.4) and (5.5). 
Case 3: The representation $\mu$ is not of real type and the multiplicity of $\mu$ in $\rho$ is larger than 1. Let $p=\frac{1}{2} l$ and $\beta=2 \alpha$ if $\mu$ is of complex type and let $p=\frac{1}{4} l$ and $\beta=4 \alpha$ if it is of quaternionic type. In both cases, we have $p \geq k+2$. In each case we define the vector $E_{a, i, \xi}$ for $a=1, \ldots, p, i=1, \ldots, \beta$ and $\xi=1, \ldots, n-m$. Then similarly we can define vectors $X^{u}$ and $Y^{u}$ for $u=1, \ldots, \beta$ and use them to define the vectors $X$ and $Y$ as in Case 1. The formulas of $P_{t}(X)$ and $P_{t}(Y)$ are obtained by Theorem 4.16 and Theorem 4.19 respectively, and the rest of the proof will follow Case 1. Note that the number of the constants $b_{i}$ 's in $X$ or $Y$ is equal to $p-1$. The existence of the vector $X_{0}$ which is orthogonal to $\mathfrak{k}^{-}$follows from the fact that $p \geq k+2$.

In the case where $\mu$ is real type, the non-vanishing terms in the sectional curvature of the 2-plane spanned by $X$ and $Y$ are computed in the following

Proposition 5.7. For the vectors $X$ and $Y$ defined in (5.6), by choosing a proper value for the constant $b_{i}$, we have

(1) there exists some $X_{0} \in \mathfrak{p}$ which is orthogonal to $\mathfrak{k}^{-}$with respect to $Q$ such that $A_{+}(X, Y)=\left(h^{2}(t)-1\right) X_{0}$;

(2) $A_{+}(X, X)=A_{+}(Y, Y)$;

(3) $Q\left(P_{t}^{\prime}(X), Y\right)=0$;

(4) $-\frac{1}{4} Q\left(P_{t}^{\prime}(X), X\right) Q\left(P_{t}^{\prime}(Y), Y\right)=-\left(d_{i_{0}} A_{\alpha}\right)^{4} h^{2}(t)\left(h^{\prime}(t)\right)^{2}$.

Proof. First we compute the endomorphism $P_{t}$ on $X$ and $Y$. From the defining equations (5.4) of $X^{u}$ and (5.5) of $Y^{u}$, we have

$$
P_{t}\left(X^{u}\right)=\sum_{i=1}^{l-1} \sum_{r=1}^{\alpha} b_{i} f_{u, r} E_{i, r, 1}+\sum_{r=1}^{\alpha} p_{u, r}(t) E_{l, r, 2}
$$

and

$$
P_{t}\left(Y^{v}\right)=\sum_{j=1}^{l-1} \sum_{s=1}^{\alpha} b_{j} f_{v, s} E_{j, s, 2}+\sum_{s=1}^{\alpha} p_{v, s}(t) E_{l, s, 1}
$$

Then

$$
\begin{aligned}
{\left[X^{u}, P_{t}\left(Y^{v}\right)\right]=} & {\left[\sum_{i=1}^{l-1} b_{i} E_{i+(u-1) l, \alpha l+1}+E_{u l, \alpha l+2},\right.} \\
& \left.\sum_{j=1}^{l-1} \sum_{s=1}^{\alpha} b_{j} f_{v, s} E_{j+(s-1) l, \alpha l+2}+\sum_{s=1}^{\alpha} p_{v, s}(t) E_{s l, \alpha l+1}\right] \\
= & \left(\sum_{i=1}^{l-1} b_{i}^{2} f_{v, u}-p_{v, u}(t)\right) E_{\alpha l+2, \alpha l+1} \\
& +\sum_{i=1}^{l-1} \sum_{s=1}^{\alpha} b_{i}\left(f_{v, s} E_{i+(s-1) l, u l}-p_{v, s}(t) E_{i+(u-1) l, s l}\right),
\end{aligned}
$$


and

$$
\begin{aligned}
{\left[P_{t}\left(X^{u}\right), Y^{v}\right]=} & {\left[\sum_{i=1}^{l-1} \sum_{r=1}^{\alpha} b_{i} f_{u, r} E_{i+(r-1) l, \alpha l+1}+\sum_{r=1}^{\alpha} p_{u, r}(t) E_{r l, \alpha l+2},\right.} \\
& \left.\sum_{j=1}^{l-1} b_{j} E_{j+(v-1) l, \alpha l+2}+E_{v l, \alpha l+1}\right] \\
= & \left(\sum_{i=1}^{l-1} b_{i}^{2} f_{u, v}-p_{u, v}(t)\right) E_{\alpha l+2, \alpha l+1} \\
& +\sum_{i=1}^{l-1} \sum_{r=1}^{\alpha} b_{i}\left(f_{u, r} E_{v l, i+(r-1) l}-p_{u, r}(t) E_{r l, i+(v-1) l}\right) .
\end{aligned}
$$

Therefore

$$
\begin{aligned}
A_{+}\left(X^{u}, Y^{v}\right)=\frac{1}{2} \sum_{i=1}^{l-1} \sum_{s=1}^{\alpha} b_{i}\left(f_{v, s} E_{i+(s-1) l, u l}+f_{u, s} E_{i+(s-1) l, v l}\right. \\
\left.\quad-p_{v, s}(t) E_{i+(u-1) l, s l}-p_{u, s}(t) E_{i+(v-1) l, s l}\right) .
\end{aligned}
$$

From the above equation, only the terms such as $E_{j+(r-1) l, w l}$ have non-zero coefficients in $A_{+}(X, Y)$, and it is denoted by $c_{j, r, w}$. From the formula (5.10) and the bilinearity of $A_{+}$, we have

$$
\begin{aligned}
c_{j, r, w} & =\frac{1}{2} b_{j}\left(\sum_{v=1}^{\alpha}\left(f_{v, r} A_{w} A_{v}-p_{v, w}(t) A_{r} A_{v}\right)+\sum_{u=1}^{\alpha}\left(f_{u, r} A_{u} A_{w}-p_{u, w} A_{u} A_{r}\right)\right) \\
(5.11) & =b_{j}\left(A_{w} \sum_{v=1}^{\alpha} f_{v, r} A_{v}-A_{r} \sum_{v=1}^{\alpha} p_{v, w}(t) A_{v}\right) .
\end{aligned}
$$

We can compute the terms in (5.11) explicitly as follows:

$$
\sum_{v=1}^{\alpha} f_{v, r} A_{v}=\sum_{v=1}^{\alpha} \sum_{i=1}^{\alpha} A_{v, i} d_{i} A_{r, i} A_{v, i_{0}}=\left(A^{\tau} A D A^{\tau}\right)_{i_{0}, r}=d_{i_{0}} A_{r, i_{0}}=d_{i_{0}} A_{r}
$$

and

$$
\begin{aligned}
\sum_{v=1}^{\alpha} p_{v, w}(t) A_{v} & =\sum_{v=1}^{\alpha}\left(a_{v} a_{w} h^{2}(t) A_{v}+f_{v, w} A_{v}-a_{v} f_{w, \alpha} A_{v}\right) \\
& =d_{i_{0}} A_{w}+\left(a_{w} h^{2}(t)-f_{w, \alpha}\right) \sum_{v=1}^{\alpha} a_{v} A_{v} \\
& =d_{i_{0}} A_{w}+a_{w}\left(h^{2}(t)-1\right) \sum_{v=1}^{\alpha} f_{v, \alpha} A_{v} \\
& =d_{i_{0}} A_{w}+d_{i_{0}} a_{w} A_{\alpha}\left(h^{2}(t)-1\right),
\end{aligned}
$$

where the first equality follows Theorem 4.11

By substituting the new expressions (5.12) and (5.13) back into the expression (5.11) of $c_{j, r, w}$, we have

$$
c_{j, r, w}=-d_{i_{0}} A_{r} A_{\alpha} a_{w} b_{j}\left(h^{2}(t)-1\right) .
$$


If $r \neq w$, then $E_{j+(r-1) l, w l}$ is orthogonal to $\mathfrak{k}^{-}$with respect to $Q$. If $r=w$, by formula (5.14) for $c_{j, r, w}, c_{j, r, r}$ is a multiple of $b_{j}$. From the assumption that the representation $\rho$ is the direct sum of $\mu$ and the embedding in (3.8), the image $\mu_{*}(v)$ (for any $v \in \mathfrak{k}^{\prime}$ ) is block-wise diagonally embedded in $\mathfrak{s o}(m)$. Hence if for some $r$, the vector $v_{r}=\sum_{j=1}^{l-1} c_{j, r, r} E_{j+(r-1) l, r l}$ is orthogonal to $\mathfrak{k}^{-}$, then all vectors $v_{q}$ are orthogonal to $\mathfrak{k}^{-}$. By Lemma 5.1 of the non-transitivity of the action $\mu\left(K^{\prime}\right)$ on the sphere $S O(l) / S O(l-1)$ and by choosing the proper values of $b_{i}, v_{r}$ is orthogonal to $\mathfrak{k}^{-}$. Therefore $A_{+}(X, Y)=\left(h^{2}(t)-1\right) X_{0}$ for some $X_{0} \in \mathfrak{p}$ which is orthogonal to $\mathfrak{k}^{-}$with respect to $Q$, and (1) is proved.

By (5.4) and (5.8), we have

$$
\begin{aligned}
{\left[X^{u}, P_{t}\left(X^{v}\right)\right]=} & {\left[\sum_{i=1}^{l-1} b_{i} E_{i+(u-1) l, \alpha l+1}+E_{u l, \alpha l+2},\right.} \\
& \left.\sum_{j=1}^{l-1} \sum_{s=1}^{\alpha} b_{j} f_{v, s} E_{j+(s-1) l, \alpha l+1}+\sum_{s=1}^{\alpha} p_{v, s}(t) E_{s l, \alpha l+2}\right] \\
= & \sum_{i \neq j, \text { or } u \neq s} b_{i} b_{j} f_{v, s} E_{j+(s-1) l, i+(u-1) l}+\sum_{s \neq u} p_{v, s}(t) E_{s l, u l} .
\end{aligned}
$$

By (5.5) and (5.9), we have the same result for $\left[Y^{u}, P_{t}\left(Y^{v}\right)\right]$; therefore $A_{+}(X, X)=$ $\left[X, P_{t}(X)\right]=\left[Y, P_{t}(Y)\right]=A_{+}(Y, Y)$, which proves the formula in (2).

Next we will prove the formulas in (3) and (4) which will finish the proof of the proposition.

By (5.8) and the differential equation (4.13) for $p_{i j}$ we have

$$
P_{t}^{\prime}\left(X^{u}\right)=\sum_{r=1}^{\alpha} p_{r, u}^{\prime}(t) E_{l, r, 2}=2 h(t) h^{\prime}(t) \sum_{r=1}^{\alpha} a_{u} a_{r} E_{l, r, 2},
$$

so

$$
\begin{aligned}
Q\left(P_{t}^{\prime}\left(X^{u}\right), Y^{v}\right) & =2 a_{u} h(t) h^{\prime}(t) \sum_{r=1}^{\alpha} a_{r}\left(\sum_{j=1}^{l-1} b_{j} Q\left(E_{l, r, 2}, E_{j, v, 2}\right)+Q\left(E_{l, r, 2}, E_{l, v, 1}\right)\right) \\
& =0,
\end{aligned}
$$

and then $Q\left(P_{t}^{\prime}(X), Y\right)=0$.

By taking the inner product with $X^{v}$ instead of $Y^{v}$, we have

$$
\begin{aligned}
Q\left(P_{t}^{\prime}\left(X^{u}\right), X^{v}\right) & =2 a_{u} h(t) h^{\prime}(t) \sum_{r=1}^{\alpha} a_{r}\left(\sum_{j=1}^{l-1} b_{j} Q\left(E_{l, r, 2}, E_{j, v, 1}\right)+Q\left(E_{l, r, 2}, E_{l, v, 2}\right)\right) \\
& =2 a_{u} a_{v} h(t) h^{\prime}(t) .
\end{aligned}
$$

Therefore

$$
\begin{aligned}
Q\left(P_{t}^{\prime}(X), X\right) & =2\left(\sum_{u, v=1}^{\alpha} A_{u} A_{v} a_{u} a_{v}\right) h(t) h^{\prime}(t)=2\left(\sum_{u=1}^{\alpha} A_{u} a_{u}\right)^{2} h(t) h^{\prime}(t) \\
& =2\left(d_{i_{0}} A_{\alpha}\right)^{2} h(t) h^{\prime}(t),
\end{aligned}
$$

where the last equality follows either from (5.12) or (5.13). Similarly for $Q\left(P_{t}^{\prime}(Y), Y\right)$ and hence (4) follows, which finishes the proof. 
Remark 5.15. In the introduction, we pointed out that there are unknown but interesting cases when $m \geq k+2$ and $n=m+1$. The minimal dimension of these manifolds is 15 when $k=2, m=5$ and $n=6$. This manifold has cohomology ring different from the two 15 dimensional symmetric spaces $\mathbb{S}^{15}$ and $S O(8) /(S O(5) \times$ $S O(3))$. The geometry of these examples will be studied in another paper.

\section{Cohomogeneity one manifolds for $G=U(n)$ and $S p(n)$}

In this section, we will generalize our examples to the cases where $G=U(n)$ and $S p(n)$. First let us state the theorem in each case.

Theorem 6.1. Let $K^{\prime} / H^{\prime}=\mathbb{S}^{k}$ with $k \geq 2$ and $\rho: K^{\prime} \longrightarrow U(m)$ be a faithful representation. Suppose $\rho$ contains a class one representation $\mu: K^{\prime} \longrightarrow U(l)$ of the pair $\left(K^{\prime}, H^{\prime}\right)$ with $2 l \geq k+2$ and the multiplicity of $\mu$ in $\rho$ is 1 . For any integer $n \geq m+2$, set $G=U(n)$ and

$$
\begin{aligned}
K^{-} & =\rho\left(K^{\prime}\right) \times U(n-m) \subset U(m) \times U(n-m) \subset U(n), \\
K^{+} & =\rho\left(H^{\prime}\right) \times U(n-m+1) \subset U(m-1) \times U(n-m+1) \subset U(n), \\
H & =\rho\left(H^{\prime}\right) \times U(n-m) \subset U(n) .
\end{aligned}
$$

Then the cohomogeneity one manifold $M$ defined by the groups $H \subset\left\{K^{-}, K^{+}\right\} \subset G$ does not admit a $G$ invariant metric with non-negative sectional curvature.

Remark 6.3. Case (2) in Proposition A.4 lists the complex class one representations which have dimension smaller than $\frac{1}{2}(k+2)$. It shows that if $\operatorname{mul}(\mu, \rho)=1$, then only the defining representations of $S U(l), U(l), S p(l)$ and $S p(l) \times U(1)$ are excluded by the above theorem. The cohomogeneity one manifolds from these representations are equivariantly diffeomorphic to the homogeneous spaces $U(n+1) / \Phi\left(K^{\prime}\right) \times U(n-l+1)$ where $\Phi: K^{\prime} \longrightarrow U(l)$ is the defining representation, so they carry non-negatively curved metrics.

Similar to Lemma 5.1 in the orthogonal case, we have the following lemma:

Lemma 6.4. Assume that $K^{\prime}, H^{\prime}$ and $\mu$ are as in Theorem 6.1 with $2 l \geq k+2$. Then $\mu\left(K^{\prime}\right)$ does not act transitively on $\mathbb{C P}^{l-1}=U(l) /(U(l-1) \times U(1))$.

Proof. From the classification of the transitive actions on complex projective spaces (see $\mathrm{Be} 2$, p. 195), we only need to check the pair $(S U(2), U(1))$ for $\mathbb{C P}^{1},(U(n)$, $U(n-1))$ for $\mathbb{C P}^{n-1}$ and $(S p(n), S p(n-1))$ for $\mathbb{C P}^{2 n-1}$. In the first case, the subgroup $U(1) \subset S U(2)$ does not fix any vector in $\mathbb{C}^{2}$. In the last two cases, we have $2 l=k+1$ which contradicts the assumption on $l$. Therefore the action of $\mu\left(K^{\prime}\right)$ on $\mathbb{C P}^{l-1}$ is non-transitive.

Sketch of the Proof of Theorem 6.1. Suppose $\rho$ has the decomposition $\tau \oplus \mu$ and $\mu$ is not equivalent to any of the subrepresentations in $\tau$. Let $c(t)$ be the normal geodesic connecting the two singular orbits $B_{ \pm}=G / K^{ \pm}$with $c(0)=p_{-} \in B_{-}$and $c(L)=p_{+} \in B_{+}$. Similar to the orthogonal case, the Weyl group is isomorphic to $\mathbb{Z}_{2} \times \mathbb{Z}_{2}$ and the generators have the following representatives:

$$
w_{+}=\operatorname{diag}\left\{I_{m-1},-1, I_{n-m}\right\}
$$

and

$$
w_{-}=\operatorname{diag}\left\{A_{1}, A_{2}, \varepsilon, I_{n-m}\right\} \text { or } \operatorname{diag}\left\{A_{1}, \varepsilon I_{l}, I_{n-m}\right\},
$$

where $A_{1} \in U(r), A_{2} \in U(l-1)$ and $\varepsilon= \pm 1$. 
In addition to the matrices $\left\{E_{i, j}\right\}_{1<i \neq j \leq n}$, let $F_{i, j}$ be the symmetric matrix with $\imath=\sqrt{-1}$ in the $i, j-$ and $j, i$-entries if $i \neq j$ and $\sqrt{2} \imath$ in the $i, i$-entry if $i=j$. Then $\left\{E_{i, j}\right\}$ and $\left\{F_{i, j}\right\}$ form an orthonormal basis of the Lie algebra $\mathfrak{u}(n)$ of $U(n)$ with $Q=-\frac{1}{2} \Re \operatorname{Tr}$, where $\Re$ takes the real part of a complex number. Without loss of generality, we may assume that $r=0$, i.e., $\rho=\mu$ and then $m=l$. Let $\mathfrak{p}$ be the orthogonal complement of the Lie algebra $\mathfrak{h}$ of $H$ in the Lie algebra $\mathfrak{g}=\mathfrak{u}(n)$ of $G$ and $\mathfrak{q}$ be the subspace of $\mathfrak{p}$ spanned by the vectors $\left\{E_{a, i}\right\}$ and $\left\{F_{a, i}\right\}$ for $a=1, \ldots, m$ and $i=m+1, \ldots, n$.

Let $h^{2}(t)=g\left(E_{m, m+1}^{*}, E_{m, m+1}^{*}\right)_{c(t)}$ and then we may assume that $h(0)=1$. From Schur's lemma and Wilking's Rigidity Theorem, we have

Proposition 6.5. Suppose that the metric $g$ is non-negatively curved. Then we have

(1) $P\left(E_{a, i}\right)=E_{a, i}$ and $P\left(F_{a, i}\right)=F_{a, i}$, if $a=1, \cdots, m-1$;

(2) $P\left(E_{m, i}\right)=h^{2}(t) E_{m, i}$ and $P\left(F_{m, i}\right)=h^{2}(t) F_{m, i}$,

where $i=m+1, \ldots, n$.

From the collapsing of the Killing vector field $E_{m, m+1}^{*}$ at $p_{+}$and Weyl symmetry at $p_{-}, h(t)$ is an even function and $h(L)=0$. Let

$$
\begin{aligned}
& X=\sum_{i=1}^{m-1} b_{i} E_{i, m+1}+E_{m, m+2}+\sum_{i=1}^{m-1} c_{i} F_{i, m+1}+F_{m, m+2}, \\
& Y=\sum_{j=1}^{m-1} b_{j} E_{j, m+2}+E_{m, m+1}+\sum_{j=1}^{m-1} c_{j} F_{j, m+2}+F_{m, m+1},
\end{aligned}
$$

where $\sum_{i=1}^{m-1}\left(b_{i}^{2}+c_{i}^{2}\right)=2$.

A computation shows that $[X, Y]=0$ and results in the following Claim:

Claim. For properly chosen values of the constants $b_{i}$ and $c_{i}$, we have

(1) there exists some $X_{0} \in \mathfrak{p}$ which is orthogonal to $\mathfrak{k}^{-}$, with respect to $Q$ such that $A_{+}(X, Y)=\left(h^{2}(t)-1\right) X_{0}$

(2) $A_{+}(X, X)=A_{+}(Y, Y)$

(3) $Q\left(P_{t}^{\prime}(X), Y\right)=0$;

(4) $-\frac{1}{4} Q\left(P_{t}^{\prime}(X), X\right) Q\left(P_{t}^{\prime}(Y), Y\right)=-4\left(h(t) h^{\prime}(t)\right)^{2}$.

The existence of $X_{0}$ follows from the non-transitivity of the $\mu\left(K^{\prime}\right)$ action on $\mathbb{C P}^{l-1}=U(l) /(U(l-1) \times U(1))$ proved in Lemma 6.4. The same argument as in the orthogonal case shows that the non-negativity of the sectional curvature of the 2-plane spanned by $X^{*}$ and $Y^{*}$ gives the desired contradiction. This completes the proof in the unitary case.

Finally we discuss the case where $G=S p(n)$.

Theorem 6.6. Let $K^{\prime} / H^{\prime}=\mathbb{S}^{k}$ with $k \geq 2$ and $\rho: K^{\prime} \longrightarrow S p(m)$ be a faithful representation. Suppose $\rho$ contains a class one representation $\mu: K^{\prime} \longrightarrow S p(l)$ of the pair $\left(K^{\prime}, H^{\prime}\right)$ with $4 l \geq k+2$ and the multiplicity of $\mu$ in $\rho$ is 1 . For any integer $n \geq m+2$, set $G=S p(n)$ and

$$
\begin{aligned}
K^{-} & =\rho\left(K^{\prime}\right) \times S p(n-m) \subset S p(m) \times S p(n-m) \subset S p(n), \\
K^{+} & =\rho\left(H^{\prime}\right) \times S p(n-m+1) \subset S p(m-1) \times S p(n-m+1) \subset S p(n), \\
H & =\rho\left(H^{\prime}\right) \times S p(n-m) \subset S p(n) .
\end{aligned}
$$


Then the cohomogeneity one manifold $M$ defined by the groups $H \subset\left\{K^{-}, K^{+}\right\} \subset G$ does not admit a $G$ invariant metric with non-negative sectional curvature.

Remark 6.8. Case (3) in Proposition A.4 lists the quaternionic class one representations which have dimension smaller than $\frac{1}{4}(k+2)$. It shows that only the standard representation of $S p(l)$ for the pair $(S p(l), S p(l-1))$ is excluded by the above theorem. The cohomogeneity one manifold from this representation has a non-negatively curved metric since it is equivariantly diffeomorphic to the homogeneous space $S p(n+1) / S p(l) \times S p(n-l+1)$.

We have the following result on quaternionic projective spaces which is analogous to Lemma 5.1 and Lemma 6.4

Lemma 6.9. Assume that $K^{\prime}, H^{\prime}$ and $\mu$ are as in Theorem 6.6 with $4 l \geq k+2$. Then $\mu\left(K^{\prime}\right)$ does not act transitively on $\mathbb{H} \mathrm{P}^{l-1}=S p(l) /(S p(l-1) \times S p(1))$.

Proof. From the classification of the transitive actions on $\mathbb{H}^{l-1}$ (see [Be2], p. 195), we have $\mu\left(K^{\prime}\right)=S p(l)$ and then $H^{\prime}=S p(l-1) ; \mu$ is the standard representation of $K^{\prime}=S p(l)$. However in this case $k=4 l-1$, which contradicts the assumption $4 l \geq k+2$.

Sketch of the Proof of Theorem 6.6. The proof follows as in the complex case where $G=U(n)$ step by step. Suppose $\{1, \imath, \jmath, \kappa\}$ is the basis of $\mathbb{H}$ over the reals such that $\imath^{2}=\jmath^{2}=\kappa^{2}=-1$ and $\kappa=\imath \jmath$. Let $G_{i, j}$ denote the symmetric matrix with 1 in the $i, j-$ and $j, i$-entries if $i \neq j$, and $\sqrt{2}$ in the $i, i$-entries. Thus $\left\{E_{i, j}, \imath G_{i, j}, \jmath G_{i, j}, \kappa G_{i, j}\right\}$ forms an orthonormal basis of the Lie algebra $\mathfrak{s p}(n)$ of $\operatorname{Sp}(n)$ with $Q=-\frac{1}{2} \Re \operatorname{Tr}$. Without loss of generality, we may assume that $r=0$, i.e., $m=l$.

Let $h^{2}(t)=g\left(E_{m, m+1}^{*}, E_{m, m+1}^{*}\right)_{c(t)}$ and we may assume that $h(0)=1$. For the endomorphism $P_{t}$, one proves the following proposition which is similar to the orthogonal and complex cases.

Proposition 6.10. Suppose that the metric $g$ is non-negatively curved. Then we have

(1) $P\left(E_{a, i}\right)=E_{a, i}$ and $P\left(\theta G_{a, i}\right)=\theta G_{a, i}$, if $a=1, \cdots, m-1$;

(2) $P\left(E_{m, i}\right)=h^{2}(t) E_{m, i}$ and $P\left(\theta G_{m, i}\right)=h^{2}(t) \theta G_{m, i}$,

where $i=m+1, \ldots, n$ and $\theta$ can be one of $\imath, \jmath$ and $\kappa$.

Furthermore, $h(t)$ is an even function with $h(L)=0$. To get the desired contradiction we choose the vectors $X$ and $Y$ as follows:

$$
\begin{aligned}
& X=\sum_{i=1}^{m-1} b_{i} E_{i, m+1}+E_{m, m+2}+\sum_{i=1}^{m-1} c_{i} G_{i, m+1}+c G_{m, m+2}, \\
& Y=\sum_{j=1}^{m-1} b_{j} E_{j, m+2}+E_{m, m+1}+\sum_{j=1}^{m-1} c_{j} G_{j, m+2}+c G_{m, m+1},
\end{aligned}
$$

where $c=\imath+\jmath+\kappa$, the $b_{i}$ 's are real numbers and the $c_{i}$ 's are pure quaternionic numbers, i.e. the real part is zero. These constants satisfy the equation $1-c^{2}-$ $\sum_{i=1}^{l-1}\left(b_{i}^{2}-c_{i}^{2}\right)=0$.

A computation shows that $[X, Y]=0$ and the following Claim:

Claim. For properly chosen values of the constants $b_{i}$ and $c_{i}$, we have 
(1) there exists some $X_{0} \in \mathfrak{p}$ which is orthogonal to $\mathfrak{k}^{-}$, with respect to $Q$ such that $A_{+}(X, Y)=\left(h^{2}(t)-h^{2}(0)\right) X_{0}$;

(2) $A_{+}(X, X)=A_{+}(Y, Y)$;

(3) $Q\left(P_{t}^{\prime}(X), Y\right)=0$;

(4) $-\frac{1}{4} Q\left(P_{t}^{\prime}(X), X\right) Q\left(P_{t}^{\prime}(Y), Y\right)=-16\left(h(t) h^{\prime}(t)\right)^{2}$.

The existence of $X_{0}$ follows from the non-transitivity of the $\mu\left(K^{\prime}\right)$ action on $\mathbb{H} \mathrm{P}^{l-1}$ proved in Lemma 6.9. The contradiction now follows as in the orthogonal case.

\section{Appendix A. Class one Representations of spherical group pairs}

In this appendix, we give the classification of all class one representations of spherical pairs and list some relevant properties. The first easy step is the reduction to the case when the action on the sphere is almost effective. For the proofs and details, see [He1 or He2. All representations are considered over the complex numbers except in Proposition A.4

The classification of transitive and effective action on spheres by connected compact Lie groups is well known; see for example page 179 in Be1]. Using representation theory of compact Lie groups, it is easy to extend the classification to the almost effective case that has the following cases:

- $U(n) / U(n-1)_{m}=\mathbb{S}^{2 n-1}(n \geq 1$ and $m \in \mathbb{Z})$,

- $S p(n) \times U(1) /(S p(n-1) \times U(1))_{m}=\mathbb{S}^{4 n-1}(n \geq 1$ and $m \in \mathbb{Z}, m \neq 0)$.

For the first one, let $m \in \mathbb{Z}$, and then $A \in U(n)$ acts on $\mathbb{C}^{n}$ via $z \mapsto(\operatorname{det} A)^{m} A . z$. It induces a transitive action on $\mathbb{S}^{2 n-1} \subset \mathbb{C}^{n}$, and the isotropy subgroup at $z=$ $(1,0, \cdots, 0)^{\top}$ is

$U(n-1)_{m}=\left\{\operatorname{diag}(a, B) \in U(n) \mid a^{m+1}=(\operatorname{det} B)^{-m}, a \in U(1)\right.$ and $\left.B \in U(n-1)\right\}$.

If $n=1$, then $U(1)$ acts on the circle $\mathbb{S}^{1}$ and the isotropy subgroup is $\mathbb{Z}_{m+1}(m \neq-1)$ and it gives us all almost effective actions on the circle. In Table 1 we only list the case when the $U(1)$ action on $\mathbb{S}^{1}$ is effective, and the corresponding results in the almost effective case easily follows.

TABle 1. Complex class one representations of spherical pairs

\begin{tabular}{|c|c|c|c|c|}
\hline$K$ & $H$ & $\rho$ & & $n$ \\
\hline \hline$S O(n)$ & $S O(n-1)$ & $a \varpi_{1}$ & $a \geq 1$ & $n \geq 3$ \\
\hline$S U(n)$ & $S U(n-1)$ & $a \varpi_{1}+b \varpi_{n-1}$ & $a+b \geq 1$ & $n \geq 3$ \\
\hline$U(n)$ & $U(n-1)_{m}$ & $\begin{array}{c}a e_{1}-b e_{n}+m(a-b) \\
\left(e_{1}+\cdots+e_{n}\right)\end{array}$ & $a+b \geq 1$ & $n \geq 2$ \\
\hline$S p(n)$ & $S p(n-1)$ & $a \varpi_{1}+b \varpi_{2}$ & $a+b \geq 1$ & $n \geq 1$ \\
\hline \hline$S p(n) \times S p(1)$ & $S p(n-1) \times S p(1)$ & $\left(a \varpi_{1}+b \varpi_{2}\right) \otimes a \varpi_{1}$ & $a+b \geq 1$ & $n \geq 1$ \\
\hline$S p(n) \times U(1)$ & $S p(n-1) \times U(1)_{m}$ & $\left(a \varpi_{1}+b \varpi_{2}\right) \otimes \phi^{k}$ & $(\mathrm{~S})$ & $n \geq 1$ \\
\hline \hline$G_{2}$ & $S U(3)$ & $a \varpi_{1}$ & $a \geq 1$ & \\
\hline $\operatorname{Spin}(7)$ & $G_{2}$ & $a \varpi_{3}$ & $a \geq 1$ & \\
\hline $\operatorname{Spin}(9)$ & $S p i n(7)$ & $a \varpi_{1}+b \varpi_{4}$ & $a+b \geq 1$ & \\
\hline \hline$U(1)$ & $\{1\}$ & $\phi^{k}$ & $k \neq 0$ & \\
\hline
\end{tabular}


Similarly in the second case the group $S p(n) \times U(1)$ has different transitive actions on $\mathbb{S}^{4 n-1}$. For each $m \in \mathbb{Z},(A, z) \in S p(n) \times U(1)$ acts on $\mathbb{H}^{n}$ via $q \mapsto A$. $q z^{m}$. The isotropy subgroup at $q=(0, \cdots, 0,1)^{\top}$ is

$S p(n-1) \times U(1)_{m}=\left\{\left(\operatorname{diag}\left(A, z^{-m}\right), z\right) \in S p(n) \times U(1) \mid z \in U(1)\right.$ and $\left.A \in S p(n-1)\right\}$.

For each spherical pair $(K, H)$ with $K / H=\mathbb{S}^{n-1}$, the defining representation $\Phi: K \longrightarrow S O(n)$ is of class one. If the pair is $(S O(n), S O(n-1))$, then the class one representations are well known. They consist of the irreducible representations on the space of homogeneous harmonic polynomials. The class one representations of the spherical pairs are closely related to these representations as stated in the following

Theorem A.1. The representation $\mu$ is a class one representation of the spherical pair $(K, H)$ if and only if $\mu$ is in the decomposition of $\operatorname{Res}_{K}^{S O(n)} \rho$, where $\rho$ is a class one representation of the pair $(S O(n), S O(n-1))$ and $K$ is viewed as a subgroup of $S O(n)$ via the representation $\Phi$.

Next we consider the classification of class one representations. We list the fundamental weights for classical groups as follows.

$$
\begin{aligned}
S O(n): & \varpi_{1}=e_{1}, \cdots, \varpi_{k-1}=e_{1}+e_{2}+\cdots+e_{k-1}, \\
& \varpi_{k}=\frac{1}{2}\left(e_{1}+\cdots+e_{k}\right), n=2 k+1, \\
& \varpi_{1}=e_{1}, \cdots, \varpi_{k-1}=\frac{1}{2}\left(e_{1}+\cdots+e_{k-1}-e_{k}\right), \\
& \varpi_{k}=\frac{1}{2}\left(e_{1}+\cdots+e_{k}\right), n=2 k, \\
S U(n): & \varpi_{1}=e_{1}, \cdots, \varpi_{n-1}=e_{1}+\cdots+e_{n-1}, \text { with } e_{1}+\cdots+e_{n}=0, \\
S p(n): & \varpi_{1}=e_{1}, \cdots, \varpi_{n}=e_{1}+\cdots+e_{n} .
\end{aligned}
$$

The exceptional Lie group $G_{2}$ has two fundamental weights: $\varpi_{1}$ which is the highest weight of the 7 dimensional representation and $\varpi_{2}$ which is 14 dimensional.

The group $U(n)$ has a finite cover $S U(n) \times U(1)$, and hence its irreducible representations can be written as $\mu \otimes \phi^{k}$ where $\mu$ is an irreducible representation of $S U(n)$ and $\phi^{k}(k \in \mathbb{Z})$ is the 1 dimensional representation of $U(1): v \mapsto z^{k} v$ for any $z \in U(1)$. Hence an irreducible representation $\rho$ of $U(n)$ with highest weight $a_{1} e_{1}+\ldots+a_{n} e_{n}\left(a_{1} \geq \ldots \geq a_{n}\right)$ is the tensor product of an irreducible representation $\mu$ of $S U(n)$ and $\phi^{k}$ of $U(1)$ where $\mu$ has highest weight $\left(a_{1}-a_{n}\right) e_{1}+\ldots+\left(a_{n-1}-a_{n}\right) e_{n-1}$ and $k=-\left(a_{1}+\ldots+a_{n}\right)$. Note that the standard representation of $U(n)$ on $\mathbb{C}^{n}$, i.e., matrix multiplication from the left, has the highest weight $-e_{n}$.

The classification is given by the following

Theorem A.2. For each almost effective spherical pair $(K, H)$, Table 1 gives the classification of all complex irreducible class one representations $\rho$. In this table,

(1) the multiplicity of the trivial representation of $H$ in $\operatorname{Res}_{H}^{K}(\rho)$ is equal to 1 except for the pair $(S p(n), S p(n-1))$, where it is a 1 if $\rho$ has the highest weight $a \varpi_{1}+b \varpi_{2}$;

(2) the numbers $a$ and $b$ are non-negative integers and $k$ is an integer;

(3) in the pair $\left(S p(n) \times U(1), S p(n-1) \times U(1)_{m}\right), a, b$ and $k$ satisfy further restriction, denoted by (S): $a+b \geq 1,|m|$ divides $k$, $a$ and $\frac{k}{|m|}$ have the same parity and $\frac{|k|}{|m|} \leq a$. For other pairs, the restrictions are specified in the table; 
(4) if $n=1$ for the $S p(n)$ factor, then there does not exist $\varpi_{2}$ in $\rho$, i.e., $b=0$.

In the following we list properties of class one representations which are used in this paper.

Proposition A.3. The type and non-trivial kernel $Z$ of each complex class one representation is listed in Table 2 .

In Table 2, for $K=U(n)(a=b)$ and $K=S p(n) \times U(1)(k=0)$, the $\mathbb{Z}_{0}$ factor in the non-trivial kernel should be interpreted as the group $U(1)=\{z \cdot \operatorname{Id} \mid z \in U(1)\}$. Furthermore, $\mathbb{Z}_{2} \subset K$ is generated by $-\mathrm{Id}$ and $\mathbb{Z}_{l} \subset S U(n)$ (or $U(n)$ ) is generated by $\varphi_{l} \cdot \operatorname{Id}$ with $\varphi_{l}=\exp \left(\frac{2 \pi \sqrt{-1}}{l}\right)$. The notation $\operatorname{gcd}(p, q)$ stands for the greatest common divisor of $p, q$.

TABLE 2. Type and non-trivial kernel of class one representations

\begin{tabular}{|c|c|c|c|}
\hline$K$ & $\rho$ & Type & $Z$ \\
\hline$\overline{S S O(n)}$ & $a \varpi_{1}$ & real & $\begin{array}{l}\mathbb{Z}_{2}: \text { if both } n \\
\text { and } a \text { are even }\end{array}$ \\
\hline$S U(n)$ & $a \varpi_{1}+b \varpi_{n-1}$ & $\begin{array}{l}\text { real: if } a=b \\
\text { complex: otherwise }\end{array}$ & $\mathbb{Z}_{l}, l=\operatorname{gcd}(a+b, n)$ \\
\hline$U(n)$ & $\begin{array}{c}a e_{1}-b e_{n} \\
+m(a-b) \\
\left(e_{1}+\cdots+e_{n}\right)\end{array}$ & $\begin{array}{l}\text { real: if } a=b \\
\text { complex: otherwise }\end{array}$ & $\begin{array}{l}\mathbb{Z}_{|(a-b)(1+m n)|} \\
\text { if } a \neq b\end{array}$ \\
\hline$S p(n)$ & $a \varpi_{1}+b \varpi_{2}$ & $\begin{array}{l}\text { real: } a \text { is even } \\
\text { quaternionic: } \\
\text { otherwise }\end{array}$ & $\mathbb{Z}_{2}:$ if $a$ is even \\
\hline $\bar{~} S p(n) \times S p(1)$ & $\begin{array}{c}\left(a \varpi_{1}+b \varpi_{2}\right) \\
\otimes a \varpi_{1}\end{array}$ & real & $\begin{array}{l}\mathbb{Z}_{2} \times \mathbb{Z}_{2}: \text { if } a \neq 0 \\
\text { is even } \\
\mathbb{Z}_{2} \times S p(1) \text { : } \\
\text { if } a=0\end{array}$ \\
\hline$S p(n) \times U(1)$ & $\begin{array}{c}\left(a \varpi_{1}+b \varpi_{2}\right) \\
\otimes \phi^{k}\end{array}$ & $\begin{array}{l}\text { complex: } k \neq 0 \\
\text { real: } k=0 \\
\text { and } a \text { is even } \\
\text { quaternionic: } \\
\text { otherwise }\end{array}$ & $\begin{array}{l}\text { Id } \times \mathbb{Z}_{|k|}: \\
\text { if } a \text { is odd } \\
\mathbb{Z}_{2} \times \mathbb{Z}_{|k|}: \\
\text { if } a \text { is even }\end{array}$ \\
\hline$\overline{G_{2}}$ & $\overline{a \varpi_{1}}$ & real & 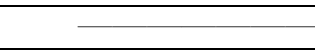 \\
\hline $\operatorname{Spin}(7)$ & $a \varpi_{3}$ & real & $\mathbb{Z}_{2}:$ if $a$ is even \\
\hline $\operatorname{Spin}(9)$ & $a \varpi_{1}+b \varpi_{4}$ & real & $\mathbb{Z}_{2}:$ if $b$ is even \\
\hline$\overline{U(1)}$ & $\overline{\phi^{k}}$ & complex & $\mathbb{Z}_{|k|}:$ if $|k|>1$ \\
\hline
\end{tabular}

Proposition A.4. Let $(K, H)$ be a spherical pair and $\rho: K \longrightarrow S O(l), U(l)$ or $S p(l)$ the embedding.

(1) Table 3 is the list of the dimension $s$ of the sphere $K / H$ and the embedding $\rho: K \longrightarrow S O(l)$ with $l \leq k_{0}(s+1)$ where $k_{0}$ is equal to 1,2 or 4 if $\rho$ is of real, complex or quaternionic type.

(2) Table 4 is the list of the dimension $s$ of the sphere $K / H$ and the embedding $\rho: K \longrightarrow U(l)$ with $2 l \leq s+1$. 
(3) The quaternionic class one representations $\rho: K \longrightarrow S p(l)$ with $4 l \leq$ $\operatorname{dim}(K / H)+1$ are exactly the representation $\rho=\varpi_{1}$ for the pair $(\operatorname{Sp}(n)$, $S p(n-1))(n \geq 1)$.

TABLE 3. Orthogonal class one representation with small dimension

\begin{tabular}{|c|c|c|c|c|c|}
\hline$K$ & $H$ & $\rho$ & $l$ & $s+1$ & $k_{0}(s+1)$ \\
\hline$\overline{S O}(n)$ & 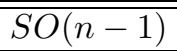 & $\overline{\varpi_{1}}$ & 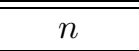 & 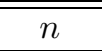 & \\
\hline$S U(n)$ & $S U(n-1)$ & {$\left[\varpi_{1}\right]_{\mathbb{R}}$} & $2 n$ & $2 n$ & $4 n$ \\
\hline$U(n)$ & $U(n-1)_{m}$ & {$\left[e_{1}+m\left(e_{1}+\cdots+e_{n}\right)\right]_{\mathbb{R}}$} & $2 n$ & $2 n$ & $4 n$ \\
\hline$S p(n)$ & $S p(n-1)$ & {$\left[\varpi_{1}\right]_{\mathbb{R}}$} & $4 n$ & $4 n$ & $16 n$ \\
\hline $\begin{array}{l}S p(n) \\
\times S p(1)\end{array}$ & $\begin{array}{l}S p(n-1) \\
\times S p(1)\end{array}$ & $\varpi_{1} \otimes \varpi_{1}$ & 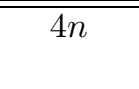 & 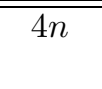 & \\
\hline $\begin{array}{l}S p(n) \\
\times U(1)\end{array}$ & $\begin{array}{l}S p(n-1) \\
\times U(1)_{m}\end{array}$ & {$\left[\varpi_{1} \otimes \phi^{k}\right]_{\mathbb{R}}$} & $4 n$ & $4 n$ & $8 n$ or $16 n$ \\
\hline$\overline{G_{2}}$ & 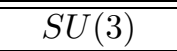 & $\overline{\varpi_{1}}$ & $\overline{7}$ & $\overline{7}$ & \\
\hline $\operatorname{Spin}(7)$ & $G_{2}$ & $\varpi_{3}$ & 8 & 8 & \\
\hline $\operatorname{Spin}(9)$ & $\operatorname{Spin}(7)$ & $\varpi_{1}, \varpi_{4}$ & 9,16 & 16 & \\
\hline$U(2)$ & $\overline{U(1)_{m}}$ & $e_{1}-e_{2}$ & 3 & 4 & \\
\hline$S p(2)$ & $S p(1)$ & $\varpi_{2}$ & 5 & 8 & \\
\hline$S p(1)$ & $\{1\}$ & $2 \varpi_{1}$ & 3 & 4 & \\
\hline$\overline{U U(1)}$ & $\{1\}$ & {$\left[\phi^{k}\right]_{\mathbb{R}}$} & 2 & 2 & $\overline{4}$ \\
\hline$U(2)$ & $U(1)_{m}$ & $\begin{array}{l}{\left[2 e_{1}+2 m\left(e_{1}+e_{2}\right)\right]_{\mathbb{R}}} \\
{\left[3 e_{1}+3 m\left(e_{1}+e_{2}\right)\right]_{\mathbb{R}}}\end{array}$ & $\begin{array}{l}6 \\
8\end{array}$ & 4 & 8 \\
\hline$U(3)$ & $U(2)_{m}$ & {$\left[2 e_{1}+2 m\left(e_{1}+e_{2}+e_{3}\right)\right]_{\mathbb{R}}$} & 12 & 6 & 12 \\
\hline$S U(3)$ & $S U(2)$ & {$\left[2 \varpi_{1}\right]_{\mathbb{R}}$} & 12 & 6 & 12 \\
\hline$\overline{S p(2)}$ & $S p(1)$ & {$\left[\varpi_{1}+\varpi_{2}\right]_{\mathbb{R}}$} & 32 & 8 & 32 \\
\hline$S p(1)$ & $\{1\}$ & {$\left[3 \varpi_{1}\right]_{\mathbb{R}},\left[5 \varpi_{1}\right]_{\mathbb{R}},\left[7 \varpi_{1}\right]_{\mathbb{R}}$} & $8,12,16$ & 4 & 16 \\
\hline
\end{tabular}

TABle 4. Complex class one representation with small dimension

\begin{tabular}{|c|c|c|c|c|}
\hline$K$ & $H$ & $\rho$ & $l$ & $s+1$ \\
\hline \hline$S U(n)$ & $S U(n-1)$ & $\varpi_{1}, \varpi_{n-1}$ & $n, n$ & $2 n$ \\
\hline$U(n)$ & $U(n-1)$ & $e_{1},-e_{n}$ & $n, n$ & $2 n$ \\
\hline$S p(n)$ & $S p(n-1)$ & $\varpi_{1}$ & $2 n$ & $4 n$ \\
\hline$S p(n) \times U(1)$ & $S p(n-1) \times U(1)_{m}$ & $\varpi_{1} \otimes \phi^{k}$ & $2 n$ & $4 n$ \\
\hline
\end{tabular}

\section{ACKNOWLEDGEMENTS}

This paper is part of the author's Ph.D. thesis at the University of Pennsylvania. The author wants to thank his advisor, Professor Wolfgang Ziller, for his generous support and great patience, and Professor Kristopher Tapp for valuable discussions on Wilking's rigidity results. 


\section{REFERENCES}

[AA] A. V. Alekseevskiǔ and D. V. Alekseevskiı̆, G-manifolds with one-dimensional orbit space, Lie groups, their discrete subgroups, and invariant theory, Adv. Soviet Math., vol. 8, Amer. Math. Soc., Providence, RI, 1992, pp. 1-31. MR.1155662 (93c:57029)

[Be1] Arthur L. Besse, Einstein manifolds, Ergebnisse der Mathematik und ihrer Grenzgebiete (3) [Results in Mathematics and Related Areas (3)], vol. 10, Springer-Verlag, Berlin, 1987. MR867684 (88f:53087)

[Be2] Arthur L. Besse, Manifolds all of whose geodesics are closed, Ergebnisse der Mathematik und ihrer Grenzgebiete [Results in Mathematics and Related Areas], vol. 93, SpringerVerlag, Berlin, 1978. With appendices by D. B. A. Epstein, J.-P. Bourguignon, L. BérardBergery, M. Berger and J. L. Kazdan. MR496885 (80c:53044)

[Ch] Jeff Cheeger, Some examples of manifolds of nonnegative curvature, J. Differential Geometry 8 (1973), 623-628. MR0341334 (49 \#6085)

[GWZ] Karsten Grove, Burkhard Wilking, and Wolfgang Ziller, Positively curved cohomogeneity one manifolds and 3-Sasakian geometry, J. Differential Geom. 78 (2008), no. 1, 33-111. MR2406265 (2009m:53090)

[GVWZ] Karsten Grove, Luigi Verdiani, Burkhard Wilking, and Wolfgang Ziller, Non-negative curvature obstructions in cohomogeneity one and the Kervaire spheres, Ann. Sc. Norm. Super. Pisa Cl. Sci. (5) 5 (2006), no. 2, 159-170. MR2244696(2007h:53051)

[GZ1] Karsten Grove and Wolfgang Ziller, Curvature and symmetry of Milnor spheres, Ann. of Math. (2) 152 (2000), no. 1, 331-367, DOI 10.2307/2661385. MR1792298(2001i:53047)

[GZ2] Karsten Grove and Wolfgang Ziller, Cohomogeneity one manifolds with positive Ricci curvature, Invent. Math. 149 (2002), no. 3, 619-646, DOI 10.1007/s002220200225. MR.1923478 (2004b:53052)

[He1] Chenxu He, Non-negatively curved cohomogeneity one manifolds, ProQuest LLC, Ann Arbor, MI, 2009. Thesis (Ph.D.)-University of Pennsylvania. MR 2713860

[He2] C. He, New examples of obstructions to non-negative sectional curvatures in cohomogeneity one manifolds, arXiv math:DG/0910. 5712v1, 2009.

[St] Eldar Straume, Compact connected Lie transformation groups on spheres with low cohomogeneity. I, Mem. Amer. Math. Soc. 119 (1996), no. 569, vi+93. MR.1297539 (96f:57036)

[VK] N. Ja. Vilenkin and A. U. Klimyk, Representation of Lie groups and special functions. Vol. 1, Mathematics and its Applications (Soviet Series), vol. 72, Kluwer Academic Publishers Group, Dordrecht, 1991. Simplest Lie groups, special functions and integral transforms. Translated from the Russian by V. A. Groza and A. A. Groza. MR 1143783 (93h:33009)

[Wa] Nolan R. Wallach, Minimal immersions of symmetric spaces into spheres, Symmetric spaces (Short Courses, Washington Univ., St. Louis, Mo., 1969-1970), Dekker, New York, 1972, pp. 1-40. Pure and Appl. Math., Vol. 8. MR0407774 (53 \#11545)

[Wi] Burkhard Wilking, A duality theorem for Riemannian foliations in nonnegative sectional curvature, Geom. Funct. Anal. 17 (2007), no. 4, 1297-1320, DOI 10.1007/s00039-0070620-0. MR 2373019 (2009a:53047)

[Zi] Wolfgang Ziller, Examples of Riemannian manifolds with non-negative sectional curvature, Surveys in differential geometry. Vol. XI, Surv. Differ. Geom., vol. 11, Int. Press, Somerville, MA, 2007, pp. 63-102. MR2408264 (2009h:53080)

Department of Mathematics, University of Pennsylvania, Philadelphia, Pennsylvania 19104

Current address: Department of Mathematics, University of Oklahoma, Norman, Oklahoma 73019

E-mail address: che@math.ou.edu 